\title{
Target Motion Sequence Detection Using Hidden Markov Models
}

\author{
Zhengxiang $\mathrm{Ma}^{1}$, Xu Zhang ${ }^{2}$, Hongchuan $\mathrm{Wei}^{2}$, Shuping Dang ${ }^{3}$ and Rakinder \\ $\mathrm{Kalsi}^{4}$ \\ ${ }^{1}$ School of Electrical Engineering, Zhengzhou University, Zhengzhou, P.R. China, \\ 450001 \\ ${ }^{2}$ Department of Mechanical Engineering and Materials Science, Duke University, \\ Durham, USA, NC 27708-0287 \\ ${ }^{3}$ Department of Engineering Science, University of Oxford, Oxford, OX1 3PJ, UK, \\ OX1 3PJ \\ ${ }^{4}$ Department of Chemistry, University of Warwick, Coventry, UK, CV4 7AL \\ zhxma@126.com,xz70@duke.edu,weihc6@gmail.com, \\ shuping.dang@eng.ox.ac.uk, r.k.kalsi@warwick.ac.uk
}

\begin{abstract}
This paper assesses the application of Hidden Markov Models (HMMs) in obtaining the most likely sequence of distributions one target chooses in the problem of a geometric, transversal approach to optimizing the probability of tracking maneuvering targets. Several factors that might affect the performance of the HMMs are considered in this paper. These include the number of time intervals, the overlapping of distributions, the symmetry of distributions, the number of distributions per time interval, the numbers of the types of distribution the target chooses from, and the dependence of distributions between time intervals. It is shown that the effects of these factors on the performance of HMMs by comparing the outcomes of problems whose settings are the same against the factor considered in that instance.
\end{abstract}

Keywords: Sequence detection, hidden Markov models, target motion

\section{Introduction}

With the development of detection technology, an increasing high requirements of accuracy and precision are raised [1], [2]. Our paper belongs to a research area proposing the application of a machine learning method, the Hidden Markov Model, [3], [4] to an interesting problem called a geometric transversal approach to optimizing probability of detection for manoeuvring targets [5], [6]. Specifically, this paper aims to decide the most likely sequence of target motion, given a sequence of target positions. The result can be utilized to, accurately, estimate the motion of other incoming targets and to reduce computation burden, and thus, it is useful for optimizing detection probability of manoeuvring targets. The research results can be extended to many applications to reinstate their validity [7-9].

The rest of this paper is organized as follows. Section 3.1.1 mathematically formulates the problem of interest. Then, in Section 3, the relevant results are presented and discussed corresponding to all subproblems. Finally, the paper is concluded in Section 4.

\section{Problem Formulation}

In this paper, HMMs are used to determine the most likely sequence of distributions one target chooses during its motion. The target is assumed to travel in a Region-of-Interest (ROI, see Sec. 2.1). The travelling period is divided into several time intervals by manoeuvring time instants. The time interval is simply the 
period of time between two target manoeuvrings as defined later in Sec. 2.2. During each time interval, the target will travel in the same direction and at a constant velocity. It will change its direction or velocity instantly at the manoeuvring instant. The target direction or velocity for each time interval will be randomly generated from one of the distributions of direction or velocity associated with the time interval, respectively. More specifically, in this paper, the velocity and direction of the target in future position will be independent of the past state, given the current velocity and direction for the first five sub-problems. For the last sub-problem, the distributions of target direction and velocity are dependent between time intervals.

Also, it is assumed that there are relationships between the distributions that the different targets will use. In our paper, it is assumed that at every manoeuvring point, these targets will pick the same distribution. The target which is tracked during its moving in the region of interest (ROI) is referred to as the tracked target. The other targets are defined as hidden targets. The positions of the tracked target and its velocities during each time interval are provided by sensors or radars. Our goal is to use the HMMs to calculate the probability distribution of the velocity and direction chosen by the target during its movement. In other words, our goal is to learn the most likely sequence of states, given observations. Upon learning the sequence of distributions chosen by the tracked target, the probability distributions of velocities and directions of the hidden targets can be found. Researches can then use these distributions to predict the distribution of the hidden targets at any time $t$, providing the initial positions of the targets are given, so that the ideal positions to install the sensors for detection can be decided.

For the HMMs, the difference of positions of the targets between two sequential time intervals can be seen as the observations. The velocities and the directions of the targets will be treated as states. With respect to the training data, since only the second basic problem has been solved in [3], training of the HMMs has not yet commenced. The testing data are the true sequences of distributions recorded while generating the target motion.

\subsection{Region of Interest}

The ROI $\mathrm{A}$ is a subset of two-dimensional Euclidean space $\mathfrak{R}^{2}$, where targets are assumed to move at the same altitude. It is assumed that the ROI A is a square of size $L * L$, large enough to contain all sensors in the ROI without sensors' field-of-views (FOVs) overlapping with each other. Since the ROI allows sufficient distance separation between sensors and targets, the risk of sensors colliding with other sensors or targets is negligible. Sensors and targets are simplified as point masses compared to the scale of the ROI without consideration of their geometric properties.

\subsection{Targets}

The targets' movements are modeled by the same Markov chain [10]. A Markov chain is a sequence of random variables $X_{1}, X_{2}, X_{3} \ldots$ with the Markov property that given the present state, the future and past states are independent. Formally,

$$
\operatorname{Pr}\left(X_{n+1}=x \mid X_{1}=x_{1}, X_{2}=x_{2}, \cdots, X_{n}=x_{n}\right)=\operatorname{Pr}\left(X_{n+1}=x \mid X_{n}=x_{n}\right)
$$

where $\operatorname{Pr}$ denotes the probability function and the possible values of $X_{i}$ form a countable set $\mathrm{X}$, known as the state space of the chain.

Targets are assumed to move over a time interval $\left[T_{0}, T_{f}\right]$, of which the starting time

$T_{0}$ and end time $T_{f}$ are fixed. $T_{f}$ is considered as the operational lifetime of batterypowered sensor networks [11]. Let the random variables $\theta(t), v(t)$ and $x(t)$ represent the 
target's direction, velocity and position, respectively. Since the targets obey the same Markov motion model, the time interval $\left[T_{0}, T_{f}\right]$ can be divided into $m$ parts $\Delta t_{j}=\left(t_{j+1}-t_{j}\right), j=1, \ldots, m$, such that the direction and velocity during each interval are constant. $\theta_{j}$ and $v_{j}$ are adopted to represent the random variables of target's direction and velocity respectively in the $j^{\text {th }}$ time interval. Since they are constant during the $j^{\text {th }}$ time interval, $\theta(t)=\theta_{j}$ and $v(t)=v_{j}$ where $t_{j}<t<t_{j+1}$ and $j=1, \ldots, m . t_{j}, j=1, \ldots, m$ are the time instants when the target changes the direction or velocity and $t_{1}=T_{0}, t_{m+1}=T_{f}$. They are assumed to be known constants in the sense that approximate information about when the targets change their headings or velocities are obtained before deploying the sensors, but are not necessarily equally spaced. The changing of a target's direction or velocity is called a manoeuvre.

It is assumed that the dynamics of targets are constrained by the nonholonomic unicycle model [12],

$$
\dot{\mathbf{x}}_{j}(t)=\left[\begin{array}{lll}
v_{j} \cos \theta_{j} & v_{j} \sin \theta_{j}
\end{array}\right]^{T}, t_{j} \leq t<t_{j+1}, j=1, \cdots, m
$$

Figure 1 shows one example of the trajectory of a maneuvering target which is modeled by the Markov Chain and obeys the nonholonomic unicycle model.

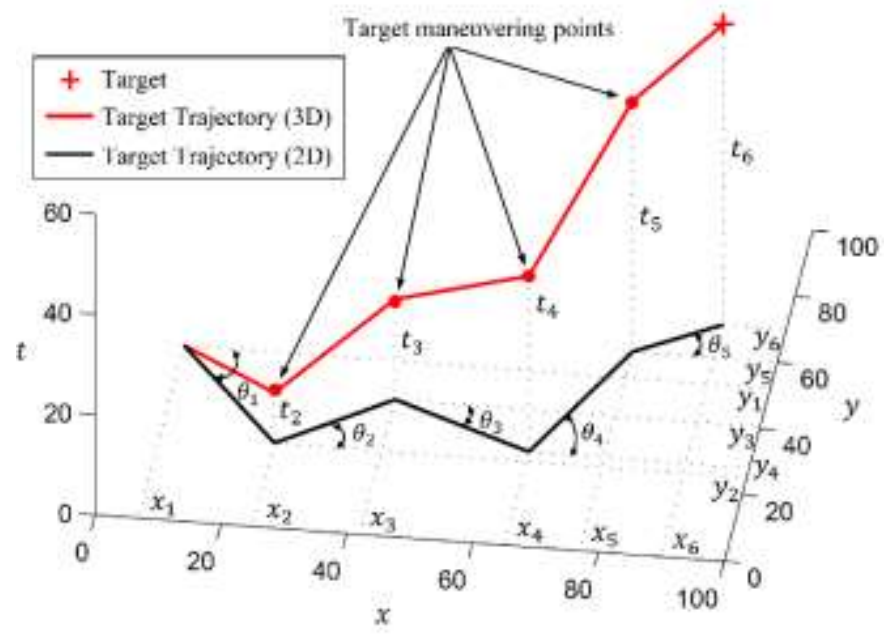

Figure 1. One Sampled Trajectory (Red) of the Target That Is Modeled By the Markov Chain and Obeys the Nonholonomic Unicycle Model. The Target Has Four Manoeuvres Represented By the Red Dots

Let $\mathbf{x}_{j}$ denote the target's initial position of the $j^{\text {th }}$ time interval, namely $\mathbf{x}_{j}=\mathbf{x}\left(t_{j}\right), j=1, \cdots, m$. Since $\theta_{j}$ and $v_{j}$ are piece-wise constant, by integrating the linear differential equation (2) over time, the set of target's initial positions is related in the way that

$$
\mathbf{x}_{j+1}=\mathbf{x}_{j}+\left[\begin{array}{ll}
v_{j} \cos \theta_{j} & v_{j} \sin \theta_{j}
\end{array}\right]^{T} \Delta t_{j}, j=1, \cdots, m
$$

Thus, the distribution of targets only depends on the random variables $\mathbf{x}(t), \theta$ and $v$ in the previous time interval and is a Markov chain. In this paper, $\theta_{j}$ and $v_{j}$ are assumed to be independent from one time interval $\left[t_{j}, t_{j+1}\right]$ to the next time interval $\left[t_{j+1}, t_{j+2}\right]$, for all 
$j=1, \cdots, m-1$. Only the target's initial position distribution $\operatorname{Pr}\left(\mathbf{x}_{j}\right)=f_{T}^{j}\left(\mathbf{x}_{j}\right)$ is a function of the random variables from the previous time interval, where $f_{T}^{j}\left(\mathbf{x}_{j}\right)$ is the probability density function (PDF) of the random variable $\mathbf{x}_{j}$. The set of random variables that affect the target's distribution in the next time interval are referred to as the Markov motion parameter $\mathrm{M}_{j}=\left\{\mathbf{x}_{j}, \theta_{j}, v_{j}\right\}, j=1, \cdots, m$. PDFs of the target's direction and velocity in each time interval are known and are denoted by $f_{\Theta}^{j}\left(\theta_{j}\right)$ and $f_{V}^{j}\left(v_{j}\right)$, respectively. The PDF of target's initial positions can be derived as follows:

$$
\begin{aligned}
& f_{T}^{j+1}\left(\mathbf{x}_{j+1}\right)=\int_{\theta_{\min }^{j}}^{\theta_{\max }^{j}} \int_{v_{\min }^{j}}^{v_{\max }^{j}} f_{T}^{j}\left(\mathbf{x}_{j}-\left[\cos \theta_{j} \sin \theta_{j}\right]^{T} v_{j} \Delta t_{j}\right) \\
& \cdot f_{\Theta}^{j}\left(\theta_{j}\right) f_{V}^{j}\left(v_{j}\right) d v_{j} d \theta_{j}, j=1, \cdots, m
\end{aligned}
$$

where $\theta_{\min }^{j}, \theta_{\max }^{j}, v_{\min }^{j}$ and $v_{\max }^{j}$ represent limits of the target's heading and velocity in the $j^{\text {th }}$ time interval, respectively.

Table 1. Velocity Distribution for Problem 0

\begin{tabular}{|c|c|c|c|l|}
\hline $\begin{array}{c}\text { Time Interval } \\
T_{1}\end{array}$ & $\begin{array}{c}\text { Velocity } \\
\text { Distribution }\end{array}$ & Probability & $\begin{array}{c}\text { Distribution } \\
\text { type }\end{array}$ & Parameter \\
\hline$T_{1}$ & $v_{11}$ & $1 / 2$ & Gaussian & $\mu_{11}=2, \sigma_{11}=0.5$ \\
\cline { 2 - 5 } & $v_{12}$ & $1 / 2$ & Gaussian & $\mu_{12}=4, \sigma_{12}=0.5$ \\
\hline$T_{2}$ & $v_{21}$ & $1 / 2$ & Gaussian & $\mu_{21}=2, \sigma_{21}=0.5$ \\
\cline { 2 - 5 } & $v_{22}$ & $1 / 2$ & Gaussian & $\mu_{22}=4, \sigma_{22}=0.5$ \\
\hline
\end{tabular}

Table 2. Heading Distribution for Problem 0

\begin{tabular}{|c|c|c|c|l|}
\hline $\begin{array}{c}\text { Time Interval } \\
T_{1}\end{array}$ & $\begin{array}{c}\text { Heading } \\
h_{11}\end{array}$ & Probability & $\begin{array}{c}\text { Distribution } \\
\text { type }\end{array}$ & Parameter \\
\hline \multirow{2}{*}{$T_{1}$} & $h_{11}$ & $1 / 2$ & Gaussian & $\mu_{11}=\pi / 4, \sigma_{11}=\pi / 4$ \\
\cline { 2 - 5 } & $h_{12}$ & $1 / 2$ & Gaussian & $\mu_{12}=\pi / 4, \sigma_{12}=\pi / 4$ \\
\hline \multirow{2}{*}{$T_{2}$} & $h_{21}$ & $1 / 2$ & Gaussian & $\mu_{21}=\pi / 3, \sigma_{21}=\pi / 3$ \\
\cline { 2 - 5 } & $h_{22}$ & $1 / 2$ & Gaussian & $\mu_{22}=-\pi / 3, \sigma_{22}=\pi / 3$ \\
\hline
\end{tabular}




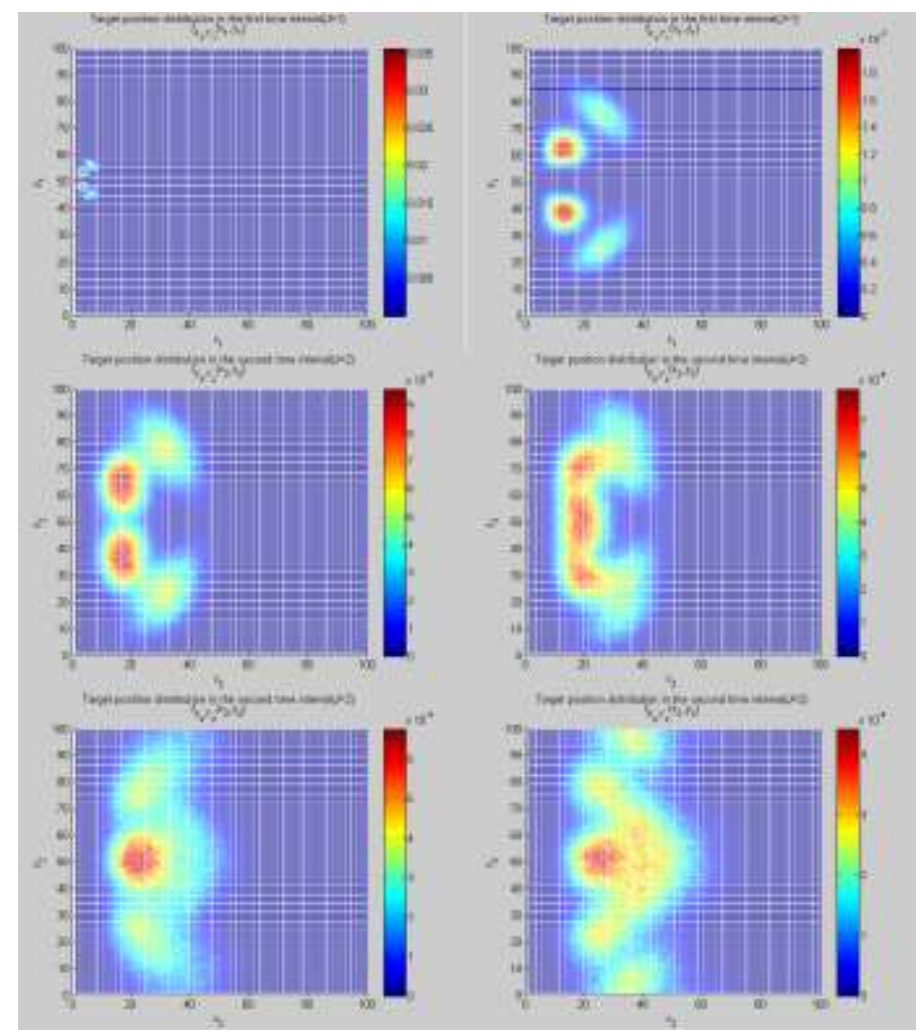

\section{Figure 2. Several Sequential Snapshots to Showcase How the Target Position Distribution Changes Over Time for Problem 0}

\subsubsection{Remark 1 on the Target}

With respect to the problem about the model of target, it is assumed the choice of velocity and direction for the current time interval does not affect the choice for the next time interval, for the first five sub-problems. The last sub-problem will be used to address the topic with respect to target model, where choices between time interval are not independent in the sense that if the target heads north in this time interval, it has more probability to head north than to head south in the next time interval. The difference between these two settings will result in different $a_{i j}$ HMMs. This paper illustrates the difference of problem settings below:

1) For the first five sub-problems

In these problems, for example, it is assumed the target direction will obey the following pattern:

- For the first time interval, it has $p=1 / 2$ probability of heading north, and $p=1 / 2$ probability of heading south;

- For the second time interval, it has $p=1 / 2$ of heading $\pi / 4$ clockwise and $p=1 / 2$ counterclockwise, etc.

In this kind of setting, the direction of target can be independent( however, it could be modeled dependently as well by changing $a_{i j}$ in the HMMs). The speed is similar to the direction, so this can also be considered independent.

2) For the last sub-problem 
For this problem, the choices of target distributions between time intervals are dependent. For example, if the target heads north in the current time interval, it will have larger probability to continue going north than to go south. The same assumption holds for the target velocity. If the target travels slowly in the current time interval, it will have larger probability to travel slowly in the next time interval.

3) Remark 2 on the Target: The target is able to change its direction and speed instantly at the maneuvering time.

\subsection{Sensors}

The omnidirectional boolean sensing model is adopted in this paper [13]. Each sensor is assumed to have a specific sensing range $r_{i}$. A sensor can only detect targets within its sensing range, where the probability for successful detection of a target entering the sensing range is 1 . In this paper, it is assumed that the sensors' positions, $\mathbf{s}_{i}=\left[s_{x_{i}} s_{y_{i}}\right]^{T}, i=1, \cdots, n$, are deterministic and static, thus the sensing range can be represented by a disk shaped FOV, $\mathrm{C}_{i}=\mathrm{C}\left[\mathbf{s}_{i}, r_{i}\right] \in \mathrm{A}$, which is centered at $\mathbf{s}_{i}$ and has a constant radius $r_{i}$. The sensing model can then be expressed as

$$
P_{d}(\mathrm{C}, \mathbf{x}(t))=\left\{\begin{array}{l}
0:\left\|\mathbf{s}_{i}-\mathbf{x}(t)\right\|>r_{i} \\
1:\left\|\mathbf{s}_{i}-\mathbf{x}(t)\right\| \leq r_{i}
\end{array}\right.
$$

\section{Results and Discussion}

\subsection{Problem 0: A Toy Problem}

This paper uses this problem and Problem 3.2 to illustrate how the number of time intervals will affect the performance of HMMs.

\subsubsection{Target Motion Assumption}

For this problem, a target has only one manoeuvre (two time intervals). It is assumed the span of the time interval is $[10,10]$. During each time interval, the target can choose from one of two direction distributions and from one of two velocity distributions. The choice of direction and velocity is independent for each time interval as explained before. Furthermore, it is assumed the choice of direction and of velocity is independent in each time interval: the direction the target chooses does not affect how fast it goes. For simplicity, it is assumed the target direction and speed obey the Gaussian distribution. The parameters of the distribution are summarized in Table 1 and Table 2. The target is assumed to start moving from $\left[\begin{array}{ll}0 & L_{2} / 2\end{array}\right]^{T}$, where $L_{2}$ is the height of the region of interest(ROI).

\subsubsection{PDF of Target Position}

As assumed in Sec. 3.1.1, the target position distribution will spread from $\left[\begin{array}{ll}0 & L_{2}\end{array}\right]^{T}$. Frames of the movie are presented to showcase the general trend in Figure 2. 
Table 3. State Vector for Problem 0

\begin{tabular}{|c|c|}
\hline $\begin{array}{c}\text { Time Interval } \\
T_{i}\end{array}$ & State vector \\
\hline$S_{1}$ & $\left(h_{11}, v_{11}\right)$ \\
$S_{2}$ & $\left(h_{11}, v_{12}\right)$ \\
$S_{3}$ & $\left(h_{12}, v_{11}\right)$ \\
$S_{4}$ & $\left(h_{12}, v_{12}\right)$ \\
\hline$S_{5}$ & $\left(h_{21}, v_{21}\right)$ \\
$S_{6}$ & $\left(h_{21}, v_{22}\right)$ \\
$S_{7}$ & $\left(h_{22}, v_{21}\right)$ \\
$S_{8}$ & $\left(h_{22}, v_{22}\right)$ \\
\hline
\end{tabular}

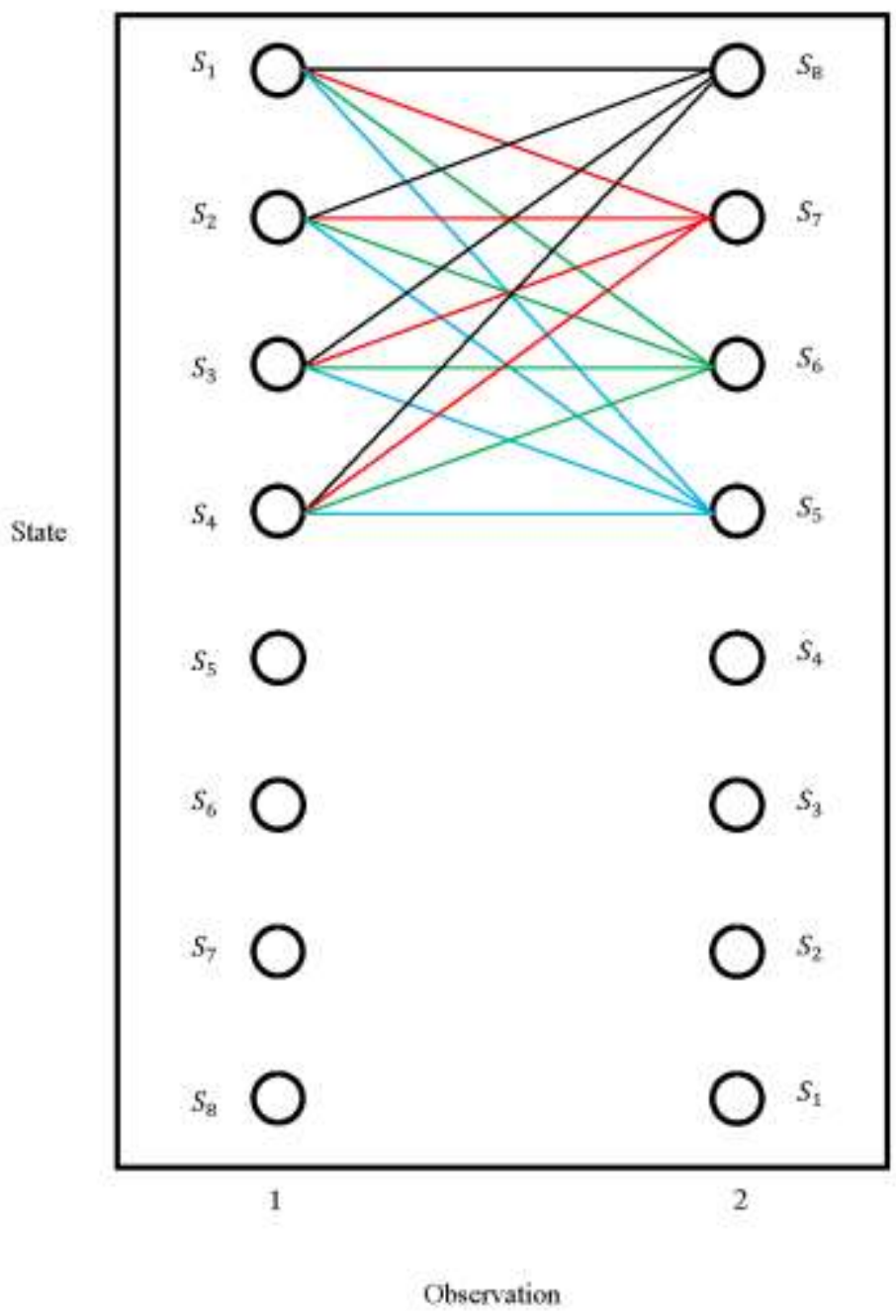

Figure 3. The Lattice of Observations and States for Problem 0 


\subsubsection{First Attempt of HMMs}

As assumed in Sec. 3.1.1, the HMMs have eight states (four for each time interval) in total, where the state of HMM is defined by the target direction and speed. Formally, a state $s_{i}$ is

$$
s_{i}=\left[h_{p q}, v_{r s}\right]
$$

where $h_{p q}$ and $v_{r s}$ denotes which distribution of target direction and speed is chosen, respectively. The states of the HMMs is summarized in Table III

Once all the states are set up, we can continue to obtain the transition matrix $A$. Graphically, the transition is shown in Figure 3. Since it is assumed that the choice between time intervals is independent, the probabilities of getting to state $s_{j}$ where $5 \leq j \leq 8$ from state $s_{i}$ where $1 \leq i \leq 4$ are the same. The property is illustrated by choosing the same colour for edges incident to $s_{j}$, where $5 \leq j \leq 8$ in Figure 3. Also, noticing that it's hard to travel back in time, thus $a_{i j}=0$ where $1 \leq i \leq 4$ and $5 \leq j \leq 8$. The edges with zero weight are not shown in Figure 3. Numerically, the value of matrix $A$ is

$$
A=\left[\begin{array}{cccccccc}
0 & 0 & 0 & 0 & \frac{1}{4} & \frac{1}{4} & \frac{1}{4} & \frac{1}{4} \\
0 & 0 & 0 & 0 & \frac{1}{4} & \frac{1}{4} & \frac{1}{4} & \frac{1}{4} \\
0 & 0 & 0 & 0 & \frac{1}{4} & \frac{1}{4} & \frac{1}{4} & \frac{1}{4} \\
0 & 0 & 0 & 0 & \frac{1}{4} & \frac{1}{4} & \frac{1}{4} & \frac{1}{4} \\
0 & 0 & 0 & 0 & 0 & 0 & 0 & 0 \\
0 & 0 & 0 & 0 & 0 & 0 & 0 & 0 \\
0 & 0 & 0 & 0 & 0 & 0 & 0 & 0 \\
0 & 0 & 0 & 0 & 0 & 0 & 0 & 0
\end{array}\right]
$$

Once the parameters of the HMMs were set, the best sequence of target motion states were speculated. First, this paper randomly generated a set of target motions and recorded which distributions of directions and speed the target chose for each time interval. The observation $O$ is defined as a vector of target direction and speed for the current time interval. This paper used the HMMs to determine best sequence of states and compared these with the true sequence of states we recorded. The results are summarized in Figure 4. From the results, it can be seen that the HMMs were accurate in determining the most probable sequence of states. In one sense, this paper chose a proper machine learning method for the action recognition problem. On the other hand, so far, only a simple problem has been worked upon we whose parameters are free of complexity. However, the accurate result implies this paper has the potential to develop this method for more complicated scenarios.

\subsection{Problem 1: Effect of the Number of Time Intervals}

Comparing the result of this problem to the Problem 3.1, this paper can determine how the number of time intervals can affect the performance of HMMs. This is in the sense that all the following factors are the same expect this paper uses $[5,10,5,5,10]$ as the new time span, 
- This paper uses the same type of distribution for target heading and velocity( Gaussian).

- The number of available distribution per time interval is still two for both the direction and the velocity.

- There is little overlapping of the PDF for heading or velocity in each time interval.

- $\quad$ The distributions of heading and speed are symmetric.

- $\quad$ Distributions between time intervals are independent.

The distributions of target direction and velocity can be found in Appendix A(Table 4 and Table 5). The state is also defined in the same pattern as in Sec. 3.1. Fifty trials are conducted for this problem and the results are presented in Figure 5. The wrongly predicted states are coloured red. Seven states are predicted wrongly out of fifty times five states. The ration of correctness is,

$$
r=\frac{250-7}{250}=0.972
$$

Comparing the result in Figure 5 to the result in Figure 4, it is obvious that increasing the number of time intervals will result in worse performance of the HMMs.

\begin{tabular}{|c|c|c|c|c|}
\hline \multirow{2}{*}{ Trial } & \multicolumn{2}{|c|}{ Test Data } & \multicolumn{2}{c|}{ HMMs results } \\
\cline { 2 - 5 } & $\mathrm{s}(\mathrm{t}=1)$ & $\mathrm{s}(\mathrm{t}=2)$ & $\mathrm{s}(\mathrm{t}=1)$ & $\mathrm{s}(\mathrm{t}=2)$ \\
\hline 1 & 1 & 8 & 1 & 8 \\
\hline 2 & 3 & 7 & 3 & 7 \\
\hline 3 & 1 & 8 & 1 & 8 \\
\hline 4 & 3 & 8 & 3 & 8 \\
\hline 5 & 1 & 6 & 1 & 6 \\
\hline 6 & 4 & 8 & 4 & 8 \\
\hline 7 & 4 & 6 & 4 & 6 \\
\hline 8 & 3 & 5 & 3 & 5 \\
\hline 9 & 2 & 7 & 2 & 7 \\
\hline 10 & 2 & 5 & 2 & 5 \\
\hline 11 & 3 & 6 & 3 & 6 \\
\hline 12 & 4 & 6 & 4 & 6 \\
\hline 13 & 1 & 5 & 1 & 5 \\
\hline 14 & 3 & 7 & 3 & 7 \\
\hline 15 & 3 & 6 & 3 & 6 \\
\hline 16 & 4 & 8 & 4 & 8 \\
\hline 17 & 3 & 6 & 3 & 6 \\
\hline 18 & 2 & 8 & 2 & 8 \\
\hline 19 & 1 & 6 & 1 & 6 \\
\hline 20 & 2 & 6 & 2 & 6 \\
\hline & & & & \\
\hline
\end{tabular}

Figure 4. Best State Sequences determined by HMMs and Real Sequences from Test Data for Twenty Different Trials for Problem 0

\subsection{Problem 2: Effect of the Overlapping of Distributions}

In this section, this paper aims to show the effect of the overlapping of distributions on the performance of the HMMs, comparing to result in Sec. 3.2. For Problem 0 and Problem 1, although this paper utilizes Gaussian distributions in our analysis, this paper carefully tuned the parameters such that, for each time interval, different direction or velocity distributions have means far apart. In other words, the area under both the PDFs(overlapping) is small. For this problem, this paper increased the overlapping area. 
For example, as shown in Fig 6, the two direction distributions in Problem 1 clearly have far away centers, however, for Problem 2, the heading distributions are mixed together.

The distributions of target directions and velocity can be found in Appendix (Table 6 and Table 7). The state is also defined in the same pattern as in Sec. 3.1. Fifty trials are conducted for this problem, and the result is in Figure 7. The wrongly predicted states are coloured red. Twenty-eight states are predicted wrongly out of fifty times five states. The ration of correctness is,

$$
r=\frac{250-28}{250}=0.888
$$

Comparing the result in Figure 7 to the result in Figure 5, it can be clearly seen that the overlapping of distributions will result in a severely worse performance of the HMMs.

\begin{tabular}{|c|c|c|c|c|c|c|c|c|c|c|}
\hline \multirow{2}{*}{ Trial } & \multicolumn{5}{|c|}{ Testing Data } & \multicolumn{5}{|c|}{ HMMs Resalt } \\
\hline & $s(t=1)$ & $s(t=2)$ & $8(t=3)$ & $s(t=4)$ & $s(t=5)$ & $s(t=1)$ & $3(t=2)$ & $s(t=3)$ & $8(\mathrm{t}=4)$ & $8(t-5)$ \\
\hline 1 & 4 & 6 & 11 & 14 & 20 & 4 & 6 & 11 & 14 & 20 \\
\hline 2 & 2 & 7 & 11 & 14 & 20 & 2 & 8 & 11 & 14 & 21) \\
\hline 3 & 3 & 8 & 12 & 15 & 19 & 3 & 8 & 12 & 15 & 19 \\
\hline 4 & 3 & 5 & 10 & 15 & 19 & 3 & 5 & 10 & 15 & 19 \\
\hline 5 & 1 & 8 & 9 & 14 & 20 & 1 & 8 & 9 & 14 & 20 \\
\hline 6 & 2 & 7 & 9 & 15 & 19 & 2 & 7 & 9 & 15 & 19 \\
\hline 7 & 3 & 8 & 12 & 15 & 17 & 3 & 8 & 12 & 15 & 17 \\
\hline 8 & 3 & 7 & 11 & 13 & 19 & 3 & 7 & 11 & 13 & 19 \\
\hline 9 & 2 & 8 & 11 & 16 & 18 & 2 & 8 & 11 & 16 & 18 \\
\hline 10 & 1 & 8 & 11 & 15 & 17 & 1 & 8 & 11 & 15 & 17 \\
\hline 11 & 2 & 6 & 10 & 14 & 20 & 1 & 6 & 10 & 14 & 20) \\
\hline 12 & 1 & 6 & 10 & 16 & 17 & 1 & 6 & 10 & 16 & 17 \\
\hline 13 & 2 & 6 & 12 & 13 & 18 & 2 & 6 & 12 & 13 & 18 \\
\hline 14 & 2 & 5 & 9 & 16 & 19 & 2 & 5 & 9 & 16 & 19. \\
\hline 15 & 4 & 6 & 9 & 13 & 17 & 4 & 6 & 9 & 13 & 17 \\
\hline 16 & 1 & 8 & 9 & 14 & 17 & 1 & 8 & 9 & 14 & 17 \\
\hline 17 & 3 & 5 & 9) & 16 & 19 & 3 & 5 & 9 & 16 & 19 \\
\hline 18 & 1 & 7 & 9 & 16 & 19 & 1 & 7 & 9 & 16 & 19 \\
\hline 19 & 3 & 7 & 11 & 14 & 19 & 3 & 8 & 11 & 14 & 19 \\
\hline 20 & 4 & 5 & 9 & 16 & 20 & 4 & 5 & 9 & 16 & 20) \\
\hline 21 & 3 & 8 & 10 & 16 & 20 & 3 & 8 & 9 & 16 & 20 \\
\hline 22 & 1 & 5 & 11 & 13 & 17 & 1 & 5 & 11 & 13 & 17 \\
\hline 23 & 2 & 5 & 12 & 13 & 17 & 2 & 5 & 12 & 13 & 17 \\
\hline 24 & 3 & 8 & 9 & 13 & 18 & 3 & 8 & 9 & 13 & 18 \\
\hline 25 & 1 & 7 & 12 & 14 & 17 & 1 & 7 & 12 & 14 & 17 \\
\hline 26 & 4 & 7 & 10 & 15 & 18 & 4 & 7 & 10 & 15 & 18 \\
\hline 27 & 2 & 8 & 9 & 14 & 18 & 2 & 8 & 9 & 14 & 18 \\
\hline 28 & 2 & 6 & 12 & 15 & 19 & 2 & 6 & 12 & 15 & 19 \\
\hline 20 & 2 & 7 & 12 & 16 & 20 & 2 & 7 & 12 & 16 & 20 \\
\hline 30 & 1 & 7 & 9 & 16 & 19 & 1 & 7 & 9 & 16 & 19 \\
\hline 31 & 2 & 5 & 11 & 16 & 19 & 2 & 5 & 11 & 16 & 19 \\
\hline 32 & 4 & 8 & 11 & 14 & 17 & 4 & 8 & 11 & 14 & 17 \\
\hline 35 & $t$ & 8 & 9 & 15 & 20 & 1 & 8 & 9 & 15 & 20 \\
\hline 34 & 1 & 6 & 9 & 13 & 17 & 1 & 6 & 9 & 15 & 17 \\
\hline 35 & 4 & 6 & 9 & 16 & 18 & 4 & 6 & 9 & 16 & 18 \\
\hline 36 & 2 & 8 & 12 & 13 & 19 & 2 & 8 & 12 & 13 & 19 \\
\hline 37 & 2 & 8 & 9 & 14 & 18 & 2 & 8 & 9 & 14 & 18 \\
\hline 38 & 3 & 5 & 9 & 15 & 18 & 3 & 5 & 9 & 15 & 18 \\
\hline 39 & I & 7 & 12 & 15 & 19 & 2 & 7 & 12 & 15 & 19 \\
\hline 40 & 4 & 7 & 11 & 13 & 17 & 4 & 7 & 11 & 13 & 17 \\
\hline 41 & 1 & 7 & 12 & 15 & 17 & 1 & 7 & 12 & 15 & 17 \\
\hline 42 & 4 & 6 & 12 & 15 & 18 & 4 & 6 & 12 & 15 & 18 \\
\hline 43 & 3 & 5 & 9 & 14 & 18 & 3 & 5 & 9 & 14 & 18 \\
\hline 4 & 4 & 8 & 10 & 14 & 19 & 4 & 8 & 10 & 14 & 19 \\
\hline 45 & $t$ & 8 & 10 & 13 & 19 & 1 & 8 & 9 & 15 & 19 \\
\hline 46 & 3 & 5 & 10 & 14 & 17 & 3 & 5 & 10 & 14 & 17 \\
\hline 47 & 2 & 3 & 10 & 14 & 18 & 2 & 5 & 9 & 14 & 18 \\
\hline 48 & 2 & 5 & 10 & 16 & 18 & 2 & 5 & 10 & 16 & 18 \\
\hline 49 & 4 & 8 & 10 & 13 & 19 & 4 & 8 & 10 & 13 & 19 \\
\hline 50 & 2 & 7 & 11 & 16 & 18 & 2 & 7 & 11 & 16 & 18 \\
\hline
\end{tabular}

Figure 5. Fifty Trials of the HMMs Prediction with Incorrectly Predictions Coloured Red for Problem 1 


\subsection{Problem 3: Effect of Symmetry of Distributions}

In this section, this paper aims to assess the effect of the symmetry of distribution on the performance of the HMMs comparing to result in Sec. 3.2. For Problem 1, the direction distribution is symmetrical with respect to the $\mathrm{x}$-axis. For this problem, the weights of direction distributions were changed in a time interval, leaving the direction distributions asymmetrical with respect to the x-axis. For example, in Figure 8, the distribution of heading is not symmetric.

The distributions of target direction and velocity can be found in Appendix (Table 8 and Table 9). The state is also defined in the same pattern as in Sec. 3.1. Fifty trials are conducted for this problem, and the result is in Figure 9. The wrongly predicted states are coloured red. Five states are predicted wrongly out of fifty times five states. The ration of correctness is,

$$
r=\frac{250-5}{250}=0.98
$$

Comparing the result in Figure 9 to the result in Figure 5, it can be seen that the symmetry of distributions does not affect performance of the HMMs much.

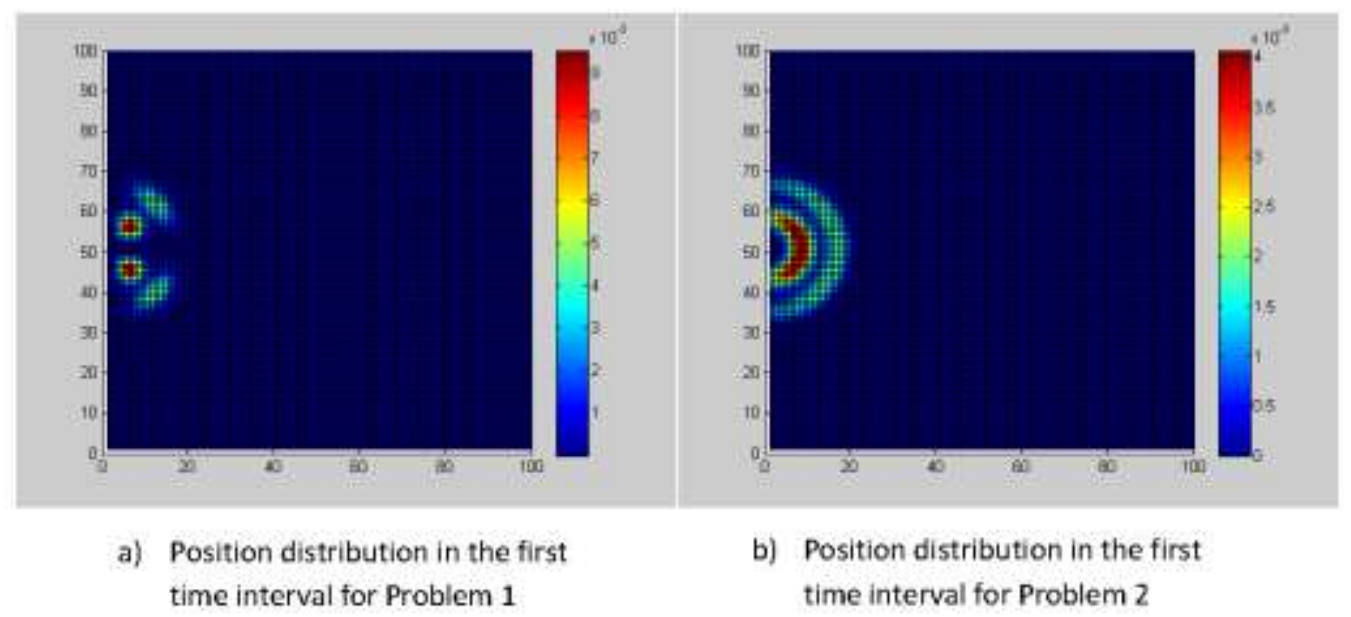

Figure 6. Position Distribution in the First Time Interval for Problem 1 and 2

\subsection{Problem 4: Effect of Type of Distributions}

In this section, the effect of the type of distribution on the performance of the HMMs is studied, comparing to result in Sec. 3.2. For Problem 1, this paper only uses Gaussian distributions for the target direction and velocity. For this problem, we use different types of distribution for target direction and speed distributions in each time interval. For example, in Figure 10, this paper can see the result of different types of distribution of direction and speed in the second time for Problem 1 and this problem.

The distributions of target direction and velocity can be found in Appendix (Table 10 and Table 11). The state is also defined in the same pattern as in Sec. 3.1. Fifty trials are conducted for this problem and the result is in Figure 11. The wrongly predicted states are coloured red. Only three states are predicted wrongly out of fifty times five states. The ration of correctness is,

$$
r=\frac{250-3}{250}=0.988
$$




\begin{tabular}{|c|c|c|c|c|c|c|c|c|c|c|}
\hline \multirow{2}{*}{ Trial } & \multicolumn{5}{|c|}{ Testing Data } & \multicolumn{5}{|c|}{ HMMs Result } \\
\hline & $s(t=1)$ & $s(t-2)$ & $\mathrm{s}(\mathrm{t}=3)$ & $s(t=4)$ & $\mathrm{s}(\mathrm{t}=5)$ & $s(t=1)$ & $\mathrm{s}(\mathrm{t}=2)$ & $s(t=3)$ & $s(t=4)$ & $s(t=5)$ \\
\hline 1 & 4 & 7 & 9 & 16 & 18 & 4 & 7 & 9 & 16 & 18 \\
\hline 2 & 4 & 8 & 12 & 13 & 17 & 4 & 8 & 12 & 13 & 17 \\
\hline 3 & 1 & 6 & 12 & 16 & 17 & 1 & 6 & 12 & 16 & 17 \\
\hline 4 & 3 & 8 & 11 & 14 & 20 & 3 & 8 & 11 & 14 & 20 \\
\hline 5 & 1 & 7 & 10 & 13 & 19 & 1 & 7 & 10 & 13 & 19 \\
\hline 6 & 3 & 8 & 9 & 13 & 17 & 3 & 8 & 11 & 13 & 17 \\
\hline 7 & 3 & 7 & 11 & 16 & 17 & 3 & 7 & 11 & 16 & 19 \\
\hline 8 & 4 & 6 & 10 & 15 & 20 & 4 & 6 & 10 & 15 & 18 \\
\hline 9 & 4 & 7 & 9 & 16 & 19 & 4 & 7 & 9 & 16 & 19 \\
\hline 10 & 3 & 5 & 11 & 13 & 17 & 3 & 5 & 11 & 15 & 19 \\
\hline 11 & 4 & 8 & 12 & 16 & 18 & 2 & 6 & 12 & 16 & 18 \\
\hline 12 & 4 & 7 & 11 & 14 & 20 & 4 & 7 & 9 & 14 & 20 \\
\hline 13 & 4 & 6 & 10 & 16 & 20 & 2 & 6 & 10 & 16 & 20 \\
\hline 14 & 4 & 8 & 9 & 15 & 19 & 4 & 8 & 9 & 15 & 19 \\
\hline 15 & 4 & 6 & 9 & 13 & 17 & 4 & 6 & 9 & 13 & 17 \\
\hline 16 & 1 & 6 & 10 & 15 & 19 & 1 & 6 & 10 & 15 & 19 \\
\hline 17 & 1 & 8 & 9 & 16 & 17 & 1 & 6 & 11 & 14 & 17 \\
\hline 18 & 3 & 5 & 10 & 15 & 20 & 3 & 5 & 10 & 15 & 20 \\
\hline 19 & 3 & 8 & 12 & 16 & 17 & 3 & 8 & 12 & 14 & 19 \\
\hline 20 & 1 & 7 & 9 & 14 & 18 & 1 & 7 & 9 & 14 & 18 \\
\hline 21 & 4 & 5 & 9 & 15 & 19 & 4 & 5 & 11 & 15 & 19 \\
\hline 22 & 4 & 8 & 9 & 14 & 19 & 4 & 8 & 9 & 14 & 19 \\
\hline 23 & 1 & 6 & 9 & 14 & 19 & 1 & 8 & 9 & 14 & 19 \\
\hline 24 & 3 & 8 & 11 & 15 & 17 & 3 & 8 & 11 & 15 & 17 \\
\hline 25 & 3 & 5 & 9 & 15 & 17 & 3 & 5 & 9 & 15 & 17 \\
\hline 26 & 1 & 7 & 9 & 13 & 20 & 1 & 7 & 9 & 13 & 20 \\
\hline 27 & 3 & 6 & 12 & 14 & 17 & 3 & 6 & 12 & 14 & 17 \\
\hline 28 & 3 & 6 & 12 & 16 & 19 & 3 & 6 & 10 & 16 & 19 \\
\hline 29 & 4 & 5 & 11 & 16 & 17 & 4 & 5 & 11 & 16 & 17 \\
\hline 30 & 3 & 8 & 11 & 13 & 20 & 3 & 8 & II & 13 & 20 \\
\hline 31 & 3 & 7 & 10 & 14 & 20 & 3 & 7 & 10 & 14 & 20 \\
\hline 32 & 3 & 8 & 11 & 13 & 20 & 3 & 8 & 11 & 13 & 20 \\
\hline 33 & 3 & 5 & 11 & 14 & 18 & 3 & 5 & 11 & 14 & 18 \\
\hline 34 & 1 & 7 & 9 & 15 & 17 & 1 & 7 & 9 & 15 & 17 \\
\hline 35 & 2 & 6 & 11 & 16 & 18 & 2 & 6 & 9 & 16 & 18 \\
\hline 36 & 2 & 5 & II & 15 & 17 & 2 & 5 & 11 & 15 & 17 \\
\hline 37 & 3 & 6 & 9 & 16 & 17 & 3 & 5 & 9 & 16 & 17 \\
\hline 38 & 1 & 6 & 12 & 14 & 17 & 1 & 6 & 10 & 16 & 17 \\
\hline 39 & 1 & 5 & 9 & 13 & 20 & 1 & 5 & 9 & 13 & 20 \\
\hline 40 & 3 & 5 & 12 & 15 & 18 & 3 & 5 & 12 & 13 & 20 \\
\hline 41 & 3 & 5 & 10 & 14 & 18 & 3 & 5 & 12 & 14 & 20 \\
\hline 42 & 1 & 6 & 11 & 15 & 20 & 1 & 6 & 11 & 15 & 20 \\
\hline 43 & 3 & 5 & 9 & 16 & 17 & 3 & 5 & 11 & 16 & 19 \\
\hline 44 & 1 & 5 & 12 & 15 & 18 & 1 & 5 & 10 & 15 & 18 \\
\hline 45 & 4 & 7 & 11 & 13 & 19 & 4 & 7 & 11 & 13 & 19 \\
\hline 46 & 2 & 8 & 11 & 16 & 19 & 2 & 6 & 11 & 16 & 19 \\
\hline 47 & 4 & 6 & 10 & 13 & 17 & 4 & 6 & 10 & 13 & 17 \\
\hline 48 & 2 & 8 & 10 & 16 & 17 & 2 & 6 & 12 & 16 & 17 \\
\hline 49 & 3 & 6 & 12 & 15 & 18 & 3 & 6 & 12 & 15 & 18 \\
\hline 50 & 2 & 8 & 12 & 15 & 18 & 2 & 8 & 12 & 15 & 18 \\
\hline
\end{tabular}

\section{Figure 7. Fifty Trials of the HMMs Prediction with Incorrectly Predictions Coloured Red for Problem 2}

Comparing the result in Figure 11 to the result in Figure 5, despite using a greater number of distributions, the performance of the HMMs is enhanced. This enhanced performance may be the result of the types of distributions. For this problem, in addition to the Gaussian distribution, this paper used uniform distribution. Also, the distributions do not overlap. Uniform distribution is more comprehensible for the HMMs, thus its performance is enhanced. 


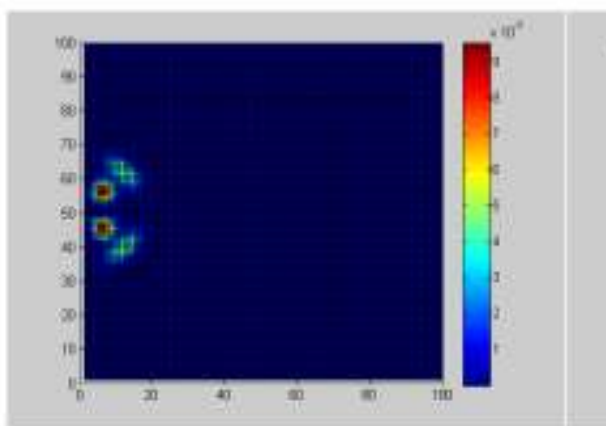

a) Position distribution in the first time interval for Problem 1

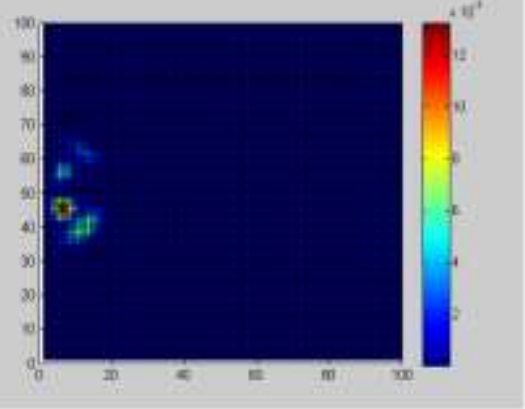

b) Position distribution in the first time interval for Problem 3

Figure 8. Position Distribution in the First Time Interval for Problem 1 and 3

\subsection{Problem 5: Effect of Number of Distributions per Time Interval}

In this section, this paper investigates the effect of the number of distributions per time interval on the performance of the HMMs comparing to result in Sec. 3.2. For Problem 1, this paper only uses two Gaussian distributions for the target direction and two Gaussian distributions for target velocity per time interval. For this problem, this paper increased the number of distributions the target direction and speed can choose from for each time interval, where the type of distribution remains the Gaussian distribution. For example, Figure 12 shows the result of different numbers of distribution of direction and speed in the second time for Problem 1 and this problem.

The distribution of target direction and velocity can be found in Appendix (Table 12 and Table 13). The state is also defined in the same pattern as in Sec. 3.1. Fifty trials are conducted for this problem, and the result is in Figure 13. The wrongly predicted states are coloured red. Seventeen states are predicted wrongly out of fifty times five states. The ration of correctness is,

$$
r=\frac{250-17}{250}=0.932
$$

Comparing the result in Figure 13 to the result in Figure 5, increasing number of states per time interval will result in worse performance of the HMMs. A reason for this is that more distributions per 


\begin{tabular}{|c|c|c|c|c|c|c|c|c|c|c|}
\hline \multirow{2}{*}{ Trial } & \multicolumn{5}{|c|}{ Testing Data } & \multicolumn{5}{|c|}{ HMEMs Result } \\
\hline & sit-1) & $4 i=2)$ & $8(t-3)$ & ब्रा(-4) & sit 5) & $40 t-11$ & 40 & $8\left(t^{-3}\right)$ & $8(1-4)$ & $s(t-5)$ \\
\hline 1 & 3 & 7 & 10 & 13 & 17 & 3 & 7 & 10 & 13 & 17 \\
\hline 2 & 2 & 8 & 12 & 14 & 18 & 2 & 8 & 12 & 14 & 18 \\
\hline 3 & 1 & 6 & 9 & 15 & 19 & 1 & 5 & 9 & 15 & 19 \\
\hline 4 & 3 & 8 & 12 & 14 & 18 & 3 & 8 & 12 & 14 & 18 \\
\hline 5 & 1 & 7 & 12 & 14 & 18 & 1 & 7 & 12 & 14 & 18 \\
\hline 6 & 3 & 7 & 10 & 16 & 20 & 3 & 7 & 10 & 16 & 20 \\
\hline 7 & 1 & 5 & 10 & 15 & 18 & 1 & 5 & 10 & 15 & 18 \\
\hline 8 & 4 & 8 & 10 & 13 & 20 & 4 & 8 & 9 & 13 & 20 \\
\hline 9 & 4 & 8 & 10 & 15 & 20 & 4 & 8 & 10 & 15 & 20 \\
\hline 10 & 4 & 8 & 9 & 13 & 18 & 4 & 8 & 9 & 13 & 18 \\
\hline 11 & 4 & 6 & 12 & 13 & 20 & 4 & 6 & 12 & 13 & 19 \\
\hline 12 & 1 & 7 & 9 & 14 & 20 & 1 & 7 & 9 & 14 & 20 \\
\hline 13 & 1 & 5 & 10 & 13 & 18 & 1 & 5 & 10 & 13 & 18 \\
\hline 14 & 3 & 7 & 10 & 15 & 17 & 3 & 7 & 10 & 15 & 17 \\
\hline 15 & 3 & 8 & 10 & 13 & 20 & 3 & 8 & 10 & 13 & 20 \\
\hline 16 & 4 & 8 & 10 & 16 & 18 & 4 & 8 & 10 & 16 & 18 \\
\hline 17 & 3 & 8 & 9 & 15 & 19 & 3 & 8 & 9 & 15 & 19 \\
\hline 18 & 4 & 7 & 12 & 15 & 18 & 4 & 7 & 12 & 15 & 18 \\
\hline 19 & 1 & 8 & 10 & 13 & 17 & 1 & 8 & 10 & 13 & 17 \\
\hline 20 & 4 & 6 & 10 & 14 & 18 & 4 & 6 & 10 & 14 & 18 \\
\hline 21 & 3 & 7 & 10 & 14 & 18 & 3 & 7 & 10 & 14 & 18 \\
\hline 22 & 4 & 8 & 11 & 14 & 19 & 4 & 8 & 11 & 14 & 19 \\
\hline 23 & 3 & 5 & 9 & 13 & 19 & 3 & 5 & 9 & 13 & 19 \\
\hline 24 & 3 & 5 & 12 & 16 & 19 & 3 & 5 & 12 & 16 & 19 \\
\hline 25 & 3 & 6 & 12 & 15 & 20 & 3 & 6 & 12 & 15 & 20 \\
\hline 26 & 2 & 8 & 11 & 14 & 18 & 2 & 8 & II & 14 & 18 \\
\hline 27 & 4 & 7 & 9 & 16 & 19 & 4 & 7 & 9 & 16 & 19 \\
\hline 28 & 2 & 8 & 11 & 16 & 18 & 2 & 8 & 11 & 16 & 18 \\
\hline 29 & 4 & 8 & 9 & 16 & 18 & 4 & 8 & 9 & 16 & 18 \\
\hline 30 & 4 & 8 & 12 & 15 & 19 & 4 & 8 & 12 & 15 & 19 \\
\hline 31 & 4 & 8 & 10 & 14 & 20 & 4 & 8 & 10 & 14 & 20 \\
\hline 32 & 3 & 6 & 12 & 16 & 18 & 3 & 6 & 12 & 16 & 18 \\
\hline 33 & 3 & 8 & 11 & 16 & 17 & 3 & 8 & 11 & 16 & 17 \\
\hline 34 & 2 & 6 & 11 & 14 & 20 & 2 & 6 & 11 & 14 & 20 \\
\hline 35 & 3 & 8 & 12 & 14 & 17 & 3 & 8 & 12 & 14 & 17 \\
\hline 36 & 2 & 7 & 9 & 16 & 17 & 2 & 8 & 9 & 16 & 17 \\
\hline 37 & 4 & 8 & 10 & 13 & 17 & 4 & 8 & 10 & 13 & 18 \\
\hline 38 & 3 & 6 & 9 & 16 & 20 & 3 & 6 & 9 & 16 & 20 \\
\hline 39 & 4 & 7 & 9 & 14 & 17 & 4 & 7 & 9 & 14 & 17 \\
\hline 40 & 3 & 8 & 12 & 13 & 20 & 3 & 8 & 12 & 13 & 20 \\
\hline 41 & 4 & 8 & 9 & 14 & 20 & 4 & 8 & 9 & 14 & 20 \\
\hline 42 & 3 & 8 & 10 & 15 & 20 & 3 & 8 & 10 & 15 & 20 \\
\hline 43 & 3 & 8 & 11 & 16 & 20 & 3 & 8 & 11 & 16 & 20 \\
\hline 44 & I & 7 & 9 & 15 & 19 & I & 7 & 9 & 15 & 19 \\
\hline 45 & 4 & 6 & 10 & 15 & 17 & 4 & 6 & 10 & 15 & 17 \\
\hline 46 & 1 & 8 & 10 & 14 & 18 & 1 & 8 & 10 & 14 & 18 \\
\hline 47 & 2 & 6 & 12 & 16 & 17 & 2 & 6 & 12 & 16 & 17 \\
\hline 48 & 4 & 7 & 10 & 14 & 17 & 4 & 7 & 10 & 14 & 17 \\
\hline 49 & 4 & 8 & 10 & 15 & 18 & 4 & 8 & 10 & 15 & 18 \\
\hline 50 & 3 & 5 & 9 & 15 & 17 & 3 & 5 & 9 & 15 & 17 \\
\hline
\end{tabular}

Figure 9. Fifty Trials of the HMMs Prediction with Incorrectly Predictions Coloured Red for Problem 3

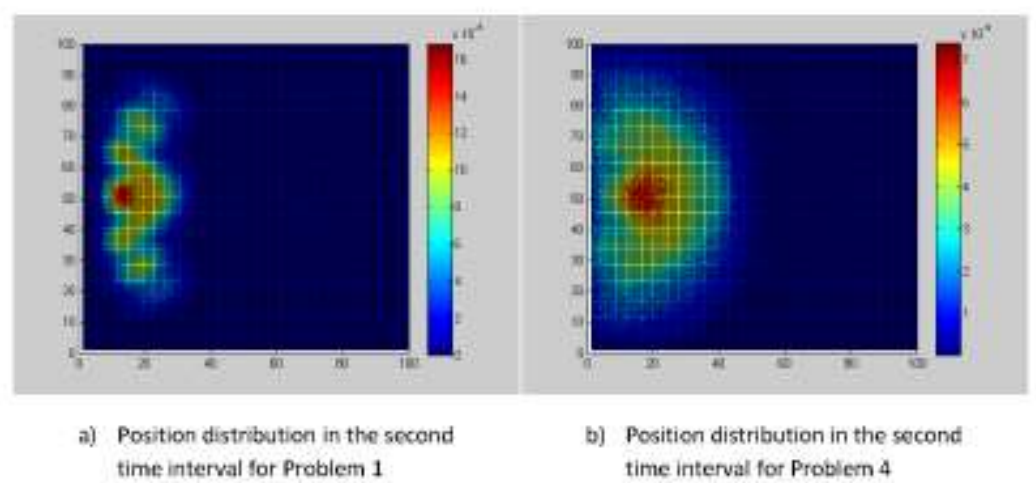

Figure 10. Position Distribution in the Second Time Interval for Problem 1 and 4 


\begin{tabular}{|c|c|c|c|c|c|c|c|c|c|c|}
\hline \multirow{2}{*}{ Irial } & \multicolumn{5}{|c|}{ Testing Data } & \multicolumn{5}{|c|}{ HMMb Reauht } \\
\hline & $3(t-1)$ & sit-2) & (s(-3) & $5(-4)$ & $2(t-5)$ & $y(-1)$ & sit $(t-2)$ & \begin{tabular}{|l}
$s i(-3)$ \\
\end{tabular} & $\mid$ ait-4) & $3(t-5)$ \\
\hline I & 1 & 5 & 9 & 15 & 17 & 1 & 5 & 9 & 15 & 17 \\
\hline 2 & 1 & 3 & 9 & 16 & 17 & 1 & 1 & 9 & 16 & 17 \\
\hline 7 & 3 & 3 & 11 & 15 & 20 & 3 & 3 & 11 & 15 & 20 \\
\hline 4 & 3 & 6 & 10 & 16 & 59 & 3 & 6 & 10 & 16 & 19 \\
\hline 3 & 2 & 7 & 9 & 14 & 17 & 2 & 7 & 9 & 14 & 17 \\
\hline 6 & 2 & 8 & 9 & 14 & 19 & $T$ & 8 & 9 & 14 & 19 \\
\hline 7 & 4 & 8 & 10. & 13 & 19 & 4 & 8 & 30 & 13 & 20 \\
\hline 8 & 1 & 7 & 9 & 16 & 18 & 1 & $\bar{T}$ & 9 & 16 & 18 \\
\hline 9 & 4 & 7 & 10 & 16 & 17 & 4 & 7 & 10 & 16 & 17 \\
\hline 10 & 3 & 3 & 9 & 15 & 19 & 3 & 3 & 9 & 15 & 19 \\
\hline II & 3 & 6 & 12 & 14 & 20 & 3 & 5 & 12 & 14 & 20 \\
\hline 12 & 1 & 8 & 10 & 14 & 18 & $T$ & 8 & 10 & 34 & 18 \\
\hline 13 & 4 & $\mathrm{~g}$ & 9 & 13 & 20 & 4 & 8 & 9 & 13 & 20 \\
\hline 14 & 2 & 8 & 12 & 14 & 19 & 2 & 8 & 12 & 14 & 19 \\
\hline 15 & 2 & 7 & 12 & 13 & 10 & 2 & 7 & 12 & 13 & 19 \\
\hline 16 & 3 & 5 & 12 & 16 & 19 & 3 & 6 & 12 & 16 & 19 \\
\hline 17 & $\frac{2}{2}$ & 7 & 12 & 15 & 17 & 2 & 7 & 12 & 15 & 17 \\
\hline 18 & 4 & 3 & 12 & 13 & 20 & 4 & 5 & 12 & 13 & 20 \\
\hline 19 & 2 & 5 & 12 & 16 & 17 & $\frac{2}{2}$ & 5 & 12 & 16 & 17 \\
\hline 20 & 2 & 8 & 12 & 15 & 17 & 2 & 8 & $n$ & 15 & 17 \\
\hline 21 & 3 & 3 & 11 & 14 & 20 & 4 & 5 & II & 14 & 20 \\
\hline 22 & 3 & 8 & $\frac{12}{12}$ & 14 & 20 & 3 & 8 & 12 & 14 & 20 \\
\hline 23 & 1 & 5 & 9 & 13 & 19 & 1 & 5 & 9 & 13 & 19 \\
\hline 21 & $I$ & 5 & 10 & 15 & 18 & 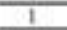 & 5 & 10 & 15 & 18 \\
\hline 23 & 1 & 8 & II & 13 & 19 & $I$ & 8 & 11 & 13 & 19 \\
\hline 8 & 4 & 5 & 9 & 15 & 18 & 4 & 5 & 9 & 15 & 18 \\
\hline 27 & 2 & 6 & 10 & 16 & 10 & 2 & 6 & 10 & 16 & 19 \\
\hline 28 & 2 & 8 & 11 & 16 & 18 & 2 & 8 & 11 & 16 & 18 \\
\hline 29 & 4 & 8 & 11 & 15 & 19 & 4 & 8 & II & 15 & 19 \\
\hline 31) & 1 & 6 & 9 & 14 & 20 & $T$ & 6 & 9 & 14 & 20 \\
\hline 31 & 3 & 8 & 11 & 14 & 20 & 3 & 8 & 11 & 34 & 20 \\
\hline 32. & 2 & 8 & 9 & 13 & 18 & 2 & 8 & 9 & 13 & 18 \\
\hline 33 & 3 & 8 & 10 & 16 & 18 & 3 & 8 & 10 & 16 & 18 \\
\hline 34 & 2 & 7 & 9 & 15 & 18 & 2 & 7 & 9 & 13 & 18 \\
\hline 35 & 3 & 8 & 9 & 14 & 19 & 3 & 8 & 9 & 14 & 19 \\
\hline 36 & 2 & 7 & 12 & 13 & 20 & 2 & 7 & 12 & 13 & 20 \\
\hline 37 & 4 & 7 & 9 & 16 & 20 & 4 & 7 & 9 & 16 & 20 \\
\hline 33 & 4 & 8 & 12 & 14 & 19 & 4 & 8 & 12 & 14 & 19 \\
\hline 39 & 3 & 8 & 10 & 14 & 17 & 3 & 8 & 10 & 14 & 17 \\
\hline 40 & 2 & 7 & 10 & 16 & 20 & 2 & 7 & 10 & 16 & 20 \\
\hline 41 & 3 & 8 & 11 & 16 & 17 & 3 & 8 & 11 & 16 & 17 \\
\hline 42 & 4 & 8 & 10 & 14 & 18 & 4 & 8 & 10 & 14 & 18 \\
\hline 43 & 1 & 3 & 9 & 14 & 19 & 1 & 5 & 9 & 14 & 19 \\
\hline 4 & 3 & 3 & 12 & 13 & 17 & 3 & 5 & 12 & 73 & 17 \\
\hline 45 & 1 & 8 & 9 & 16 & 17 & 1 & 8 & 9 & 16 & 17 \\
\hline 46 & 4 & 5 & 10 & 16 & 20 & 4 & 5 & 10 & 16 & 20 \\
\hline 47 & 1 & 6 & 12 & 14 & 10 & 1 & 6 & 12 & 14 & 19 \\
\hline 48 & 4 & 5 & 12 & 16 & 17 & 4 & 5 & 12 & 16 & 17 \\
\hline 49 & 4 & 7 & 11 & 14 & 20 & 4 & 7 & II & 14 & 20 \\
\hline का & 3 & 5 & 12 & 14 & 17 & 3 & 5 & 12 & 14 & 17 \\
\hline
\end{tabular}

Figure 11. Fifty Trials of the HMMs Prediction with Incorrectly Predictions Coloured Red for Problem 4

time interval provides more overlapping, where it has been previously shown that overlapping will cause the HMMs to perform badly. Another reason is that the searching space of HMMs will increase greatly.

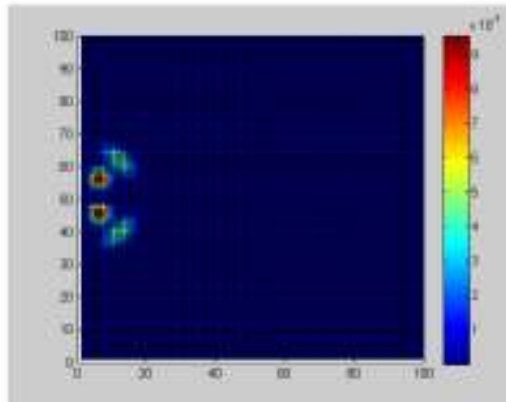

a) Position distribution in the first time interval for Problem 1

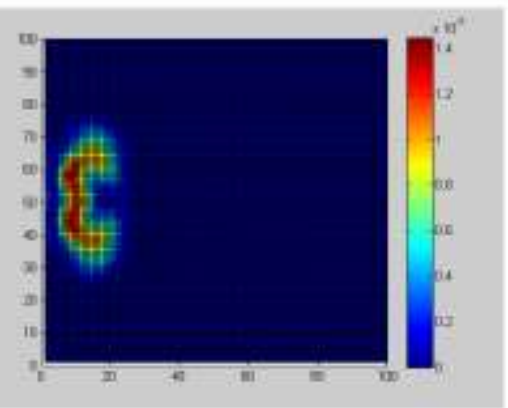

b) Position distribution in the first time interval for Problem 5

Figure 12. Position Distribution in the First Time Interval for Problem 1 and 5 


\subsection{Problem 6: Effect of Dependence of Distributions between Time Intervals}

In this section, this paper wants to show the effect of the dependence of distributions between time intervals on the performance of the HMMs comparing to result in Sec. 3.2. For Problem 1, distributions of direction and speed between time intervals are independent. For this problem, it is assumed that if the target goes north for the current time interval, it will have larger probability to go north in the next time interval. For example, in Figure 14, compared to that in Problem 1, the target position distribution shrink to the mean.

\begin{tabular}{|c|c|c|c|c|c|c|c|c|c|c|}
\hline \multirow{2}{*}{ Trial } & \multicolumn{5}{|c|}{ Testing Data } & \multicolumn{5}{|c|}{ HDMs Result } \\
\hline & $4(\mathrm{t}=1)$ & $s(\mathrm{i}=2)$ & $N(1=3)$ & $x(t=-4)$ & $5(t-5)$ & $s(t=1)$ & $5(\mathrm{t}=2)$ & $\mathrm{s}(\mathrm{t}-3)$ & $s(t=4)$ & $s(t=-5)$ \\
\hline 1 & 2 & 12 & 25 & 32 & 56 & 2 & 11 & 25 & 32 & 52 \\
\hline 2 & 4 & 10 & 24 & 30 & 54 & 4 & 10 & 24 & 30 & 54 \\
\hline 3 & 4 & 11 & 22 & 28 & 45 & 4 & 12 & 22 & 28 & 45 \\
\hline 4 & 4 & III & 23 & 26 & 52 & 4 & 11 & 23 & 26 & 52 \\
\hline 3 & 2 & 13 & 17 & 38 & 42 & 2 & 13 & 17 & 34 & 42 \\
\hline 6 & 2 & 15 & 21 & 32 & 42 & 2 & 15 & 21 & 32 & 42 \\
\hline 7 & 2 & 13 & 21 & 37 & 51 & 2 & 13 & 21 & 37 & 51 \\
\hline 8 & 4 & 15 & 22 & 32 & 55 & 4 & 16 & 22 & 32 & 55 \\
\hline 9 & 4 & 10 & 21 & 37 & 52 & 4 & 10 & 20) & 37 & $\$ 2$ \\
\hline 10 & 7 & 13 & 22 & 39 & 48 & 7 & 13 & 22 & 39 & 48 \\
\hline 11 & 8 & 13 & 17 & 31 & 48 & 8 & 13 & 17 & 31 & 48 \\
\hline 12 & 1 & 10 & 18 & 30 & 43 & 1 & 10 & 18 & 30 & 43 \\
\hline 13 & 6 & 12 & 19 & 41 & 44 & 6 & 12 & 19 & 41 & 44 \\
\hline 14 & 3 & 15 & 18 & 39 & 51 & 3 & 15 & 18 & 39 & 51 \\
\hline 15 & 1 & 14 & 18 & 32 & 53 & 1 & 14 & 18 & 32 & 53 \\
\hline 16 & 7 & 12 & 20 & 40 & 52 & 8 & 12 & 20 & 36 & 52 \\
\hline 17 & 8 & 9 & 23 & 39 & 54 & 8 & 9 & 23 & 39 & 54 \\
\hline 18 & 2 & 11 & 24 & 33 & 47 & 2 & 11 & 24 & 33 & 47 \\
\hline 19 & 1 & 12 & 17 & 32 & 45 & 1 & 12 & 17 & 32 & 45 \\
\hline 20 & 1 & 12 & 23 & 36 & 36 & 1 & 12 & 23 & 36 & 56 \\
\hline 21 & 7 & 10 & 19 & 31 & 48 & 7 & 10 & 19 & 31 & 48 \\
\hline 22 & 3 & 15 & 20 & 28 & 48 & 3 & 15 & 20 & 28 & 48 \\
\hline 23 & 2 & 13 & 23 & 36 & 42 & 2 & 13 & 23 & 36 & 42 \\
\hline 24 & 8 & 9 & 20 & 36 & 49 & 8 & 9 & 20 & 36 & 45 \\
\hline 25 & 8 & 9 & 25 & 40 & 48 & 8 & 9 & 25 & 40 & 48 \\
\hline 26 & 8 & 14 & 24 & 37 & 57 & 8 & 15 & 25 & 37 & 57 \\
\hline 27 & 8 & 9 & 17 & 27 & 48 & 8 & 9 & 17 & 27 & 48 \\
\hline 28 & 7 & 12 & 20 & 37 & 48 & 7 & 12 & 20 & 37 & 48 \\
\hline 29 & 5 & 12 & 17 & 32 & 55 & 5 & 12 & 17 & 32 & 55 \\
\hline 30 & 4 & 12 & 21 & 30 & 42 & 3 & 12 & 21 & 30 & 42 \\
\hline 31 & 7 & 15 & 25 & 27 & 56 & 7 & 13 & 24 & 27 & 56 \\
\hline 32 & 7 & 11 & 18 & 36 & 51 & 7 & 11 & 18 & 36 & 47 \\
\hline 33 & 1 & 11 & 23 & 29 & 42 & 1 & 11 & 23 & 29 & 42 \\
\hline 34 & 7 & 13 & 21 & 36 & 51 & 7 & 13 & 21 & 40 & 55 \\
\hline 35 & 2 & 11 & 23 & 38 & 57 & 2 & 11 & 23 & 38 & 57 \\
\hline 36 & 2 & 14 & 24 & 41 & 43 & 2 & 14 & 24 & 37 & 43 \\
\hline 37 & 5 & 15 & 20 & 33 & 49 & 5 & 15 & 20 & 33 & 49 \\
\hline 38 & 2 & 12 & 24 & 26 & 53 & 2 & 12 & 23 & 26 & 53 \\
\hline 39 & 2 & 14 & 21 & 29 & 51 & 2 & 14 & 21 & 29 & 51 \\
\hline 40 & 5 & 9 & 20 & 31 & 43 & 5 & 9 & 20 & 31 & 43 \\
\hline 41 & 5 & 14 & 20 & 28 & 48 & 5 & 14 & 20 & 28 & 48 \\
\hline 42 & 8 & 16 & 24 & 38 & 49 & 8 & 16 & 24 & 38 & 49 \\
\hline 43 & 7 & 14 & 20 & 30 & 47 & 7 & 14 & 20 & 30 & 47 \\
\hline 44 & 8 & 14 & 22 & 40 & 56 & 8 & 14 & 22 & 40 & 56 \\
\hline 45 & 6 & 16 & 21 & 33 & 42 & 6 & 16 & 21 & 33 & 42 \\
\hline 46 & 4 & 11 & 23 & 31 & 56 & 4 & 11 & 23 & 27 & 56 \\
\hline 47 & 3 & 16 & 25 & 28 & 54 & 3 & 16 & 25 & 28 & 54 \\
\hline 48 & 8 & 9 & 22 & 31 & 50 & 8 & 9 & 22 & 31 & 50 \\
\hline 49 & 2 & 14 & 23 & 32 & 47 & 2 & 14 & 23 & 32 & 47 \\
\hline 50 & 6 & 13 & 18 & 39 & 44 & 6 & 13 & 18 & 39 & 48 \\
\hline
\end{tabular}

\section{Figure 13. Fifty Trials of the HMMs Prediction with Incorrectly Predictions Coloured Red for Problem 6}

The distribution of target direction and velocity can be found in Appendix (Table 14 and Table 15). The state is also defined in the same pattern as in Sec. 3.1. Fifty trials are conducted for this problem, and the result is in Figure 15. The wrongly predicted states 
are coloured red. Twelve states are predicted wrongly out of fifty times five states. The ration of correctness is,

$$
r=\frac{250-12}{250}=0.952
$$

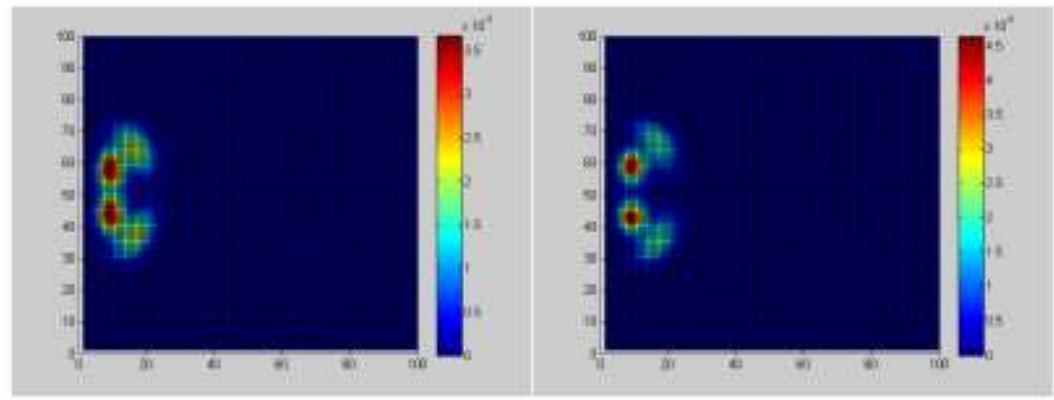

a) Position distribution in the first time interval for Problem 1

b) Position distribution in the first time interval for Problem 6

Figure 14. Position Distribution in the First Interval for Problem 1

Comparing the result in Figure 15 to the result in Figure 5, the dependency of distributions between time intervals will result in worse performance of the HMMs. Our explanation is that the problem accumulates complexity when there is dependency between time intervals. 


\begin{tabular}{|c|c|c|c|c|c|c|c|c|c|c|}
\hline \multirow{2}{*}{ Trial } & \multicolumn{5}{|c|}{ Testing Data } & \multicolumn{5}{|c|}{ HMMs Result } \\
\hline & $s(t-1)$ & $s(t-2)$ & $s(t-3)$ & $s(t-4)$ & sit-5) & $s(t-1)$ & $s(t-2)$ & $s(t-3)$ & sit-4) & $\approx(t-5)$ \\
\hline$T$ & $\overline{2}$ & 8 & 12 & 15 & 19 & 2 & 8 & 12 & 15 & 19 \\
\hline 2 & 4 & 7 & 11 & 13 & 17 & 4 & 7 & 11 & 13 & 17 \\
\hline 3 & $\mathrm{I}$ & 7 & 9 & 15 & 17 & 1 & 7 & 9 & 15 & 17 \\
\hline 4 & 2 & 6 & 10 & 14 & 18 & 2 & 6 & 10 & 14 & 18 \\
\hline 5 & 1 & 6 & 10 & 14 & 20 & 1 & 6 & 10 & 14 & 20 \\
\hline 6 & 1 & 7 & 9 & 14 & 19 & 1 & 7 & 9 & 14 & 17 \\
\hline 7 & 4 & 8 & 12 & 16 & 18 & 4 & 8 & 12 & 16 & 18 \\
\hline 8 & 2 & 5 & 12 & 16 & 18 & 2 & 5 & 12 & 14 & 18 \\
\hline 9 & 4 & 7 & 12 & 16 & 20 & 4 & 7 & 12 & 16 & 20 \\
\hline 10 & $T$ & 7 & $\pi$ & 16 & 20 & 1 & 7 & 12 & 16 & 20 \\
\hline 11 & 3 & 7 & 10 & 16 & 20 & 3 & 7 & 10 & 16 & 20 \\
\hline 12 & 3 & 7 & 9 & 13 & 17 & 3 & $\overline{7}$ & 9 & 13 & 17 \\
\hline 13 & 3 & 5 & 9 & 13 & 17 & 3 & 5 & 9 & 13 & 17 \\
\hline 14 & 4 & 6 & II & 16 & 19 & 4 & 6 & 11 & 16 & 19 \\
\hline 15 & $\frac{2}{2}$ & 6 & 9 & 14 & 19 & 2 & 6 & 9 & 14 & 19 \\
\hline 16 & 3 & 7 & 10 & 14 & 29 & 3 & 7 & 10 & 14 & 20 \\
\hline 17 & I & 5 & 9 & 15 & 17 & 1 & 5 & 9 & 15 & 17 \\
\hline 18 & 4 & 8 & 10 & 14 & 20 & 4 & 8 & 10 & 14 & 20 \\
\hline 19 & 4 & 6 & 10 & 14 & 20 & 4 & 6 & 10 & 14 & 20 \\
\hline 20 & 3 & 7 & 11 & 16 & 18 & 3 & 7 & 11 & 16 & 18 \\
\hline 21 & 3 & 5 & 11 & 15 & 20 & 3 & 5 & 11 & 15 & 20 \\
\hline 22 & 1 & 8 & 11 & 15 & 19 & 1 & 8 & 11 & 15 & 19 \\
\hline 23 & 2 & 6 & 10 & 14 & 18 & 2 & 6 & 10 & 14 & 18 \\
\hline 24 & 2 & 6 & 9 & 15 & 19 & $\frac{2}{2}$ & 6 & 9 & 13 & 19 \\
\hline 25 & 4 & 7 & 11 & 14 & 18 & 4 & 7 & 11 & 14 & 18 \\
\hline 26 & 1 & 8 & 11 & 15 & 17 & 1 & 8 & 11 & 15 & 18 \\
\hline 27 & 3 & 5 & 10 & 14 & 17 & $\sqrt{3}$ & 5 & 10 & 14 & 17 \\
\hline 28 & 3 & 5 & 9 & 14 & 17 & 3 & 5 & 9 & 14 & 17 \\
\hline 29 & 1 & 6 & 9 & 13 & 19 & 1 & 6 & 9 & 13 & 19 \\
\hline 30 & 4 & 8 & 10 & 14 & 18 & 4 & 7 & 10 & 14 & 20 \\
\hline 31 & 4 & 7 & 10 & 16 & 19 & 4 & 7 & 10 & 16 & 19 \\
\hline 32 & 3 & 7 & 9 & 13 & 17 & 3 & 7 & 9 & 13 & 17 \\
\hline 33 & 3 & 8 & 11 & 15 & 17 & 3 & 8 & 11 & 15 & 17 \\
\hline 34 & 1 & 5 & 10 & 16 & 20 & 1 & 5 & 10 & 16 & 20 \\
\hline 35 & 1 & 7 & 11 & 16 & 19 & 1 & 7 & 11 & 16 & 19 \\
\hline 36 & 4 & 6 & 12 & 16 & 19 & 4 & 6 & 12 & 16 & 20 \\
\hline 37 & 3 & 7 & 11 & 15 & 17 & 3 & 7 & 11 & 15 & 17 \\
\hline 38 & 3 & 6 & 10 & 16 & 17 & 3 & 6 & 10 & 16 & 17 \\
\hline 39 & 4 & 5 & 9 & 13 & 18 & 4 & 5 & 9 & 13 & 18 \\
\hline 40 & 3 & 7 & 11 & 16 & 211 & 3 & 7 & 11 & 16 & 10 \\
\hline 41 & 1 & 8 & 9 & 15 & 20 & 1 & 8 & 9 & 15 & 20 \\
\hline 42 & 1 & 5 & 11 & 14 & 18 & 1 & 5 & 11 & 14 & 18 \\
\hline 43 & 2 & 6 & 10 & 14 & 17 & 2 & 6 & 10 & 14 & 17 \\
\hline 44 & 2 & 6 & 12 & 15 & 20 & 2 & 5 & 12 & 15 & 20 \\
\hline 45 & 4 & $\overline{8}$ & 9 & 13 & 18 & 4 & 8 & 9 & 13 & 18 \\
\hline 46 & 1 & 6 & 12 & 16 & 20 & 1 & 6 & 12 & 16 & 20 \\
\hline 47 & 4 & 8 & 12 & 16 & 20 & 3 & 5 & 12 & 16 & 20 \\
\hline 48 & 1 & 5 & 9 & 15 & 20 & 1 & 5 & 9 & 15 & 20 \\
\hline 49 & 2 & 6 & 10 & 14 & 19 & 2 & 6 & 10 & 14 & 19 \\
\hline 50 & 1 & 7 & 9 & 13 & 18 & 1 & 7 & 9 & 13 & 18 \\
\hline
\end{tabular}

\section{Figure 15. Fifty Trials of the HMMs Prediction with Incorrectly Predictions Coloured Red for Problem 6}

\section{Conclusion}

From the comparisons this paper made, it can be concluded that:

- More time intervals will result in worse performance of the HMMs due to the larger searching space.

- Overlapping between distributions will result much worse performance of the HMMs. It is the most important factor.

- Symmetry of distributions does not have much effect on the performance.

- Number of types of distribution per time interval does not affect the performance much. However, the HMMs performs better with respect to uniform distribution than the Gaussian distribution.

- Number of distributions per time interval have an effect on the performance, due to the overlapping and increasing of searching space it may cause. 
- Dependence between distributions will result in worse performance due to more complicated problem formulation.

\section{Appendix}

Table 4. Heading Distribution for Problem 1

\begin{tabular}{|c|c|c|c|c|}
\hline $\begin{array}{c}\text { Time Interval } \\
T_{i}\end{array}$ & $\begin{array}{c}\text { Heading } \\
h_{i j}\end{array}$ & Probability & $\begin{array}{c}\text { Distribution } \\
\text { type }\end{array}$ & Parameter \\
\hline \multirow{2}{*}{$T_{1}$} & $h_{11}$ & $1 / 2$ & Gaussian & $\mu_{11}=\pi / 4, \sigma_{11}=\pi / 12$ \\
\cline { 2 - 5 } & $h_{12}$ & $1 / 2$ & Gaussian & $\mu_{12}=-\pi / 4, \sigma_{12}=\pi / 12$ \\
\hline \multirow{2}{*}{$T_{2}$} & $h_{21}$ & $1 / 2$ & Gaussian & $\mu_{21}=\pi / 3, \sigma_{21}=\pi / 12$ \\
\cline { 2 - 6 } & $h_{22}$ & $1 / 2$ & Gaussian & $\mu_{22}=-\pi / 3, \sigma_{22}=\pi / 12$ \\
\hline \multirow{2}{*}{$T_{3}$} & $h_{31}$ & $1 / 2$ & Gaussian & $\mu_{31}=\pi / 2, \sigma_{31}=\pi / 12$ \\
\cline { 2 - 6 } & $h_{32}$ & $1 / 2$ & Gaussian & $\mu_{22}=-\pi / 2, \sigma_{22}=\pi / 12$ \\
\hline \multirow{2}{*}{$T_{4}$} & $h_{41}$ & $1 / 2$ & Gaussian & $\mu_{41}=\pi / 6, \sigma_{41}=\pi / 12$ \\
\cline { 2 - 6 } & $h_{42}$ & $1 / 2$ & Gaussian & $\mu_{42}=-\pi / 6, \sigma_{42}=\pi / 12$ \\
\hline \multirow{2}{*}{$T_{5}$} & $h_{51}$ & $1 / 2$ & Gaussian & $\mu_{51}=\pi / 4, \sigma_{51}=\pi / 8$ \\
\cline { 2 - 5 } & $h_{52}$ & $1 / 2$ & Gaussian & $\mu_{52}=-\pi / 4, \sigma_{52}=\pi / 8$ \\
\hline
\end{tabular}

Table 5. Velocity Distribution for Problem 1

\begin{tabular}{|c|c|c|c|l|}
\hline $\begin{array}{c}\text { Time Interval } \\
T_{i}\end{array}$ & $\begin{array}{c}\text { Velocity } \\
\text { Distribution }\end{array}$ & Probability & $\begin{array}{c}\text { Distribution } \\
\text { type }\end{array}$ & Parameter \\
\hline \multirow{2}{*}{$T_{1}$} & $v_{11}$ & $1 / 2$ & Gaussian & $\mu_{11}=2, \sigma_{11}=0.5$ \\
\cline { 2 - 5 } & $v_{12}$ & $1 / 2$ & Gaussian & $\mu_{12}=4, \sigma_{12}=0.5$ \\
\hline \multirow{2}{*}{$T_{2}$} & $v_{21}$ & $1 / 2$ & Gaussian & $\mu_{21}=2, \sigma_{21}=0.5$ \\
\cline { 2 - 5 } & $v_{22}$ & $1 / 2$ & Gaussian & $\mu_{22}=4, \sigma_{22}=0.5$ \\
\hline \multirow{2}{*}{$T_{3}$} & $v_{31}$ & $1 / 2$ & Gaussian & $\mu_{31}=6, \sigma_{31}=0.5$ \\
\cline { 2 - 5 } & $v_{32}$ & $1 / 2$ & Gaussian & $\mu_{32}=8, \sigma_{32}=0.5$ \\
\hline \multirow{2}{*}{$T_{4}$} & $v_{41}$ & $1 / 2$ & Gaussian & $\mu_{41}=3, \sigma_{41}=0.5$ \\
\cline { 2 - 5 } & $v_{42}$ & $1 / 2$ & Gaussian & $\mu_{42}=6, \sigma_{42}=0.5$ \\
\hline \multirow{2}{*}{$T_{5}$} & $v_{51}$ & $1 / 2$ & Gaussian & $\mu_{51}=4, \sigma_{51}=0.5$ \\
\cline { 2 - 5 } & $v_{52}$ & $1 / 2$ & Gaussian & $\mu_{52}=6, \sigma_{52}=0.5$ \\
\hline
\end{tabular}


Table 6. Heading Distribution for Problem 2

\begin{tabular}{|c|c|c|c|l|}
\hline $\begin{array}{c}\text { Time Interval } \\
T_{i}\end{array}$ & $\begin{array}{c}\text { Heading } \\
h_{i j}\end{array}$ & Probability & $\begin{array}{c}\text { Distribution } \\
\text { type }\end{array}$ & Parameter \\
\hline \multirow{2}{*}{$T_{1}$} & $h_{11}$ & $1 / 2$ & Gaussian & $\mu_{11}=\pi / 4, \sigma_{11}=\pi / 4$ \\
\cline { 2 - 5 } & $h_{12}$ & $1 / 2$ & Gaussian & $\mu_{12}=-\pi / 4, \sigma_{12}=\pi / 4$ \\
\hline \multirow{2}{*}{$T_{2}$} & $h_{21}$ & $1 / 2$ & Gaussian & $\mu_{21}=\pi / 3, \sigma_{21}=\pi / 3$ \\
\cline { 2 - 5 } & $h_{22}$ & $1 / 2$ & Gaussian & $\mu_{22}=-\pi / 3, \sigma_{22}=\pi / 3$ \\
\hline \multirow{2}{*}{$T_{3}$} & $h_{31}$ & $1 / 2$ & Gaussian & $\mu_{31}=\pi / 2, \sigma_{31}=\pi / 2$ \\
\cline { 2 - 5 } & $h_{32}$ & $1 / 2$ & Gaussian & $\mu_{22}=-\pi / 2, \sigma_{22}=\pi / 2$ \\
\hline \multirow{2}{*}{$T_{4}$} & $h_{41}$ & $1 / 2$ & Gaussian & $\mu_{41}=\pi / 6, \sigma_{41}=\pi / 6$ \\
\cline { 2 - 6 } & $h_{42}$ & $1 / 2$ & Gaussian & $\mu_{42}=-\pi / 6, \sigma_{42}=\pi / 6$ \\
\hline \multirow{2}{*}{$T_{5}$} & $h_{51}$ & $1 / 2$ & Gaussian & $\mu_{51}=\pi / 4, \sigma_{51}=\pi / 4$ \\
\cline { 2 - 5 } & $h_{52}$ & $1 / 2$ & Gaussian & $\mu_{52}=-\pi / 4, \sigma_{52}=\pi / 4$ \\
\hline
\end{tabular}

Table 7. Velocity Distribution for Problem 2

\begin{tabular}{|c|c|c|c|l|}
\hline $\begin{array}{c}\text { Time Interval } \\
T_{i}\end{array}$ & $\begin{array}{c}\text { Velocity } \\
\text { Distribution }\end{array}$ & Probability & $\begin{array}{c}\text { Distribution } \\
\text { type }\end{array}$ & Parameter \\
\hline$T_{1}$ & $v_{11}$ & $1 / 2$ & Gaussian & $\mu_{11}=2, \sigma_{11}=0.5$ \\
\cline { 2 - 5 } & $v_{12}$ & $1 / 2$ & Gaussian & $\mu_{12}=4, \sigma_{12}=0.5$ \\
\hline \multirow{2}{*}{$T_{2}$} & $v_{21}$ & $1 / 2$ & Gaussian & $\mu_{21}=2, \sigma_{21}=0.5$ \\
\cline { 2 - 5 } & $v_{22}$ & $1 / 2$ & Gaussian & $\mu_{22}=4, \sigma_{22}=0.5$ \\
\hline$T_{3}$ & $v_{31}$ & $1 / 2$ & Gaussian & $\mu_{31}=4, \sigma_{31}=0.5$ \\
\cline { 2 - 5 } & $v_{32}$ & $1 / 2$ & Gaussian & $\mu_{32}=8, \sigma_{32}=0.5$ \\
\hline \multirow{2}{*}{$T_{4}$} & $v_{41}$ & $1 / 2$ & Gaussian & $\mu_{41}=3, \sigma_{41}=0.5$ \\
\cline { 2 - 5 } & $v_{42}$ & $1 / 2$ & Gaussian & $\mu_{42}=6, \sigma_{42}=0.5$ \\
\hline$T_{5}$ & $v_{51}$ & $1 / 2$ & Gaussian & $\mu_{51}=2, \sigma_{51}=0.5$ \\
\cline { 2 - 5 } & $v_{52}$ & $1 / 2$ & Gaussian & $\mu_{52}=6, \sigma_{52}=0.5$ \\
\hline
\end{tabular}

Table 8. Heading Distribution for Problem 3

\begin{tabular}{|c|c|c|c|l|}
\hline $\begin{array}{c}\text { Time Interval } \\
T_{i}\end{array}$ & $\begin{array}{c}\text { Heading } \\
h_{i j}\end{array}$ & Probability & $\begin{array}{c}\text { Distribution } \\
\text { type }\end{array}$ & Parameter \\
\hline$T_{1}$ & $h_{11}$ & 0.3 & Gaussian & $\mu_{11}=\pi / 4, \sigma_{11}=\pi / 12$ \\
\cline { 2 - 5 } & $h_{12}$ & 0.7 & Gaussian & $\mu_{12}=-\pi / 4, \sigma_{12}=\pi / 12$ \\
\hline$T_{2}$ & $h_{21}$ & 0.3 & Gaussian & $\mu_{21}=\pi / 3, \sigma_{21}=\pi / 12$ \\
\cline { 2 - 5 } & $h_{22}$ & 0.7 & Gaussian & $\mu_{22}=-\pi / 3, \sigma_{22}=\pi / 12$ \\
\hline$T_{3}$ & $h_{31}$ & 0.7 & Gaussian & $\mu_{31}=\pi / 2, \sigma_{31}=\pi / 12$ \\
\hline
\end{tabular}




\begin{tabular}{|c|c|c|c|c|}
\hline & $h_{32}$ & 0.3 & Gaussian & $\mu_{22}=-\pi / 2, \sigma_{22}=\pi / 12$ \\
\hline \multirow{2}{*}{$T_{4}$} & $h_{41}$ & 0.5 & Gaussian & $\mu_{41}=\pi / 6, \sigma_{41}=\pi / 12$ \\
\cline { 2 - 5 } & $h_{42}$ & 0.5 & Gaussian & $\mu_{42}=-\pi / 6, \sigma_{42}=\pi / 12$ \\
\hline \multirow{2}{*}{$T_{5}$} & $h_{51}$ & 0.6 & Gaussian & $\mu_{51}=\pi / 4, \sigma_{51}=\pi / 8$ \\
\cline { 2 - 5 } & $h_{52}$ & 0.4 & Gaussian & $\mu_{52}=-\pi / 4, \sigma_{52}=\pi / 8$ \\
\hline
\end{tabular}

Table 9. Velocity Distribution for Problem 3

\begin{tabular}{|c|c|c|c|l|}
\hline $\begin{array}{c}\text { Time Interval } \\
T_{i}\end{array}$ & $\begin{array}{c}\text { Velocity } \\
\text { Distribution }\end{array}$ & Probability & $\begin{array}{c}\text { Distribution } \\
\text { type }\end{array}$ & Parameter \\
\hline \multirow{2}{*}{$T_{1}$} & $v_{11}$ & $1 / 2$ & Gaussian & $\mu_{11}=2, \sigma_{11}=0.5$ \\
\cline { 2 - 5 } & $v_{12}$ & $1 / 2$ & Gaussian & $\mu_{12}=4, \sigma_{12}=0.5$ \\
\hline \multirow{2}{*}{$T_{2}$} & $v_{21}$ & $1 / 2$ & Gaussian & $\mu_{21}=2, \sigma_{21}=0.5$ \\
\cline { 2 - 5 } & $v_{22}$ & $1 / 2$ & Gaussian & $\mu_{22}=4, \sigma_{22}=0.5$ \\
\hline \multirow{2}{*}{$T_{3}$} & $v_{31}$ & $1 / 2$ & Gaussian & $\mu_{31}=6, \sigma_{31}=0.5$ \\
\cline { 2 - 5 } & $v_{32}$ & $1 / 2$ & Gaussian & $\mu_{32}=8, \sigma_{32}=0.5$ \\
\hline \multirow{2}{*}{$T_{4}$} & $v_{41}$ & $1 / 2$ & Gaussian & $\mu_{41}=3, \sigma_{41}=0.5$ \\
\cline { 2 - 5 } & $v_{42}$ & $1 / 2$ & Gaussian & $\mu_{42}=6, \sigma_{42}=0.5$ \\
\hline \multirow{2}{*}{$T_{5}$} & $v_{51}$ & $1 / 2$ & Gaussian & $\mu_{51}=4, \sigma_{51}=0.5$ \\
\cline { 2 - 5 } & $v_{52}$ & $1 / 2$ & Gaussian & $\mu_{52}=6, \sigma_{52}=0.5$ \\
\hline
\end{tabular}

Table 10. Heading Distribution for Problem 4

\begin{tabular}{|c|c|c|c|c|}
\hline $\begin{array}{c}\text { Time } \\
\text { Interval } \\
T_{i}\end{array}$ & $\begin{array}{c}\text { Heading } \\
h_{i j}\end{array}$ & Probability & $\begin{array}{c}\text { Distribution } \\
\text { type }\end{array}$ & Parameter \\
\hline$T_{1}$ & $h_{11}$ & $1 / 2$ & Gaussian & $\mu_{11}=\pi / 4, \sigma_{11}=\pi / 12$ \\
\cline { 2 - 5 } & $h_{12}$ & $1 / 2$ & Gaussian & $\mu_{12}=-\pi / 4, \sigma_{12}=\pi / 12$ \\
\hline \multirow{2}{*}{$T_{2}$} & $h_{21}$ & $1 / 2$ & uniform & $\theta_{\min }=0, \theta_{\max }=2 \pi / 3$ \\
\cline { 2 - 5 } & $h_{22}$ & $1 / 2$ & uniform & $\theta_{\min }=-2 \pi / 3, \theta_{\max }=0$ \\
\hline \multirow{2}{*}{$T_{3}$} & $h_{31}$ & $1 / 2$ & Gaussian & $\mu_{31}=\pi / 2, \sigma_{31}=\pi / 12$ \\
\cline { 2 - 5 } & $h_{32}$ & $1 / 2$ & Gaussian & $\mu_{22}=-\pi / 2, \sigma_{22}=\pi / 12$ \\
\hline \multirow{2}{*}{$T_{4}$} & $h_{41}$ & $1 / 2$ & uniform & $\theta_{\min }=0, \theta_{\max }=\pi / 3$ \\
\cline { 2 - 5 } & $h_{42}$ & $1 / 2$ & uniform & $\theta_{\min }=-\pi / 3, \theta_{\max }=0$ \\
\hline \multirow{2}{*}{$T_{5}$} & $h_{51}$ & $1 / 2$ & Gaussian & $\mu_{51}=\pi / 4, \sigma_{51}=\pi / 8$ \\
\cline { 2 - 5 } & $h_{52}$ & $1 / 2$ & uniform & $\theta_{\min }=-\pi / 2, \theta_{\max }=0$ \\
\hline
\end{tabular}


Table 11. Velocity Distribution for Problem 4

\begin{tabular}{|c|c|c|c|l|}
\hline $\begin{array}{c}\text { Time Interval } \\
T_{i}\end{array}$ & $\begin{array}{c}\text { Velocity } \\
\text { Distribution }\end{array}$ & Probability & $\begin{array}{c}\text { Distribution } \\
\text { type }\end{array}$ & Parameter \\
\hline \multirow{2}{*}{$T_{1}$} & $v_{11}$ & $1 / 2$ & Gaussian & $\mu_{11}=2, \sigma_{11}=0.5$ \\
\cline { 2 - 5 } & $v_{12}$ & $1 / 2$ & Gaussian & $\mu_{12}=4, \sigma_{12}=0.5$ \\
\hline \multirow{2}{*}{$T_{2}$} & $v_{21}$ & $1 / 2$ & uniform & $v_{\text {min }}=1, v_{\max }=3$ \\
\cline { 2 - 5 } & $v_{22}$ & $1 / 2$ & uniform & $v_{\min }=3, v_{\max }=5$ \\
\hline \multirow{2}{*}{$T_{3}$} & $v_{31}$ & $1 / 2$ & uniform & $v_{\min }=5, v_{\max }=7$ \\
\cline { 2 - 5 } & $v_{32}$ & $1 / 2$ & uniform & $v_{\min }=7, v_{\max }=9$ \\
\hline \multirow{2}{*}{$T_{4}$} & $v_{41}$ & $1 / 2$ & Gaussian & $\mu_{41}=3, \sigma_{41}=0.5$ \\
\cline { 2 - 5 } & $v_{42}$ & $1 / 2$ & Gaussian & $\mu_{42}=6, \sigma_{42}=0.5$ \\
\hline \multirow{2}{*}{$T_{5}$} & $v_{51}$ & $1 / 2$ & Gaussian & $\mu_{51}=4, \sigma_{51}=0.5$ \\
\cline { 2 - 5 } & $v_{52}$ & $1 / 2$ & uniform & $v_{\min }=7, v_{\max }=9$ \\
\hline
\end{tabular}

\begin{tabular}{|c|c|c|c|c|c|c|c|c|c|c|c|c|c|c|c|c|c|c|c|}
\hline 0 & 0 & 0 & 0 & 0.25 & 0.25 & 0.25 & 0.25 & 0 & 0 & 0 & 0 & 0 & 0 & 0 & 0 & 0 & 0 & 0 & 0 \\
\hline 0 & 0 & 0 & 0 & 0.25 & 0.25 & 0.25 & 0.25 & 0 & 0 & 0 & 0 & 0 & 0 & 0 & 0 & 0 & 0 & 0 & 0 \\
\hline 0 & 0 & 0 & 0 & 0.25 & 0.25 & 0.25 & 0.25 & 0 & 0 & 0 & 0 & 0 & 0 & 0 & 0 & 0 & 0 & 0 & 0 \\
\hline 0 & 0 & 0 & 0 & 0.25 & 0.25 & 0.25 & 0.25 & 0 & 0 & 0 & 0 & 0 & 0 & 0 & 0 & 0 & 0 & 0 & 0 \\
\hline 0 & 0 & 0 & 0 & 0 & 0 & 0 & 0 & 0.25 & 0.25 & 0.25 & 0.25 & 0 & 0 & 0 & 0 & 0 & 0 & 0 & 0 \\
\hline 0 & 0 & 0 & 0 & 0 & 0 & 0 & 0 & 0.25 & 0.25 & 0.25 & 0.25 & 0 & 0 & 0 & 0 & 0 & 0 & 0 & 0 \\
\hline 0 & 0 & 0 & 0 & 0 & 0 & 0 & 0 & 0.25 & 0.25 & 0.25 & 0.25 & 0 & 0 & 0 & 0 & 0 & 0 & 0 & 0 \\
\hline 0 & 0 & 0 & 0 & 0 & 0 & 0 & 0 & 0.25 & 0.25 & 0.25 & 0.25 & 0 & 0 & 0 & 0 & 0 & 0 & 0 & 0 \\
\hline 0 & 0 & 0 & 0 & 0 & 0 & 0 & 0 & 0 & 0 & 0 & 0 & 0.25 & 0.25 & 0.25 & 0.25 & 0 & 0 & 0 & 0 \\
\hline 0 & 0 & 0 & 0 & 0 & 0 & 0 & 0 & 0 & 0 & 0 & 0 & 0.25 & 0.25 & 0.25 & 0.25 & 0 & 0 & 0 & 0 \\
\hline 0 & 0 & 0 & 0 & 0 & 0 & 0 & 0 & 0 & 0 & 0 & 0 & 0.25 & 0.25 & 0.25 & 0.25 & 0 & 0 & 0 & 0 \\
\hline 0 & 0 & 0 & 0 & 0 & 0 & 0 & 0 & 0 & 0 & 0 & 0 & 0.25 & 0.25 & 0.25 & 0.25 & 0 & 0 & 0 & 0 \\
\hline 0 & 0 & 0 & 0 & 0 & 0 & 0 & 0 & 0 & 0 & 0 & 0 & 0 & 0 & 0 & 0 & 0.25 & 0.25 & 0.25 & 0.25 \\
\hline 0 & 0 & 0 & 0 & 0 & 0 & 0 & 0 & 0 & 0 & 0 & 0 & 0 & 0 & 0 & 0 & 0.25 & 0.25 & 0.25 & 0.25 \\
\hline 0 & 0 & 0 & 0 & 0 & 0 & 0 & 0 & 0 & 0 & 0 & 0 & 0 & 0 & 0 & 0 & 0.25 & 0.25 & 0.25 & 0.25 \\
\hline 0 & 0 & 0 & 0 & 0 & 0 & 0 & 0 & 0 & 0 & 0 & 0 & 0 & 0 & 0 & 0 & 0.25 & 0.25 & 0.25 & 0.25 \\
\hline 0 & 0 & 0 & 0 & 0 & 0 & 0 & 0 & 0 & 0 & 0 & 0 & 0 & 0 & 0 & 0 & 0 & 0 & 0 & 0 \\
\hline 0 & 0 & 0 & 0 & 0 & 0 & 0 & 0 & 0 & 0 & 0 & 0 & 0 & 0 & 0 & 0 & 0 & 0 & 0 & 0 \\
\hline 0 & 0 & 0 & 0 & 0 & 0 & 0 & 0 & 0 & 0 & 0 & 0 & 0 & 0 & 0 & 0 & 0 & 0 & 0 & 0 \\
\hline 0 & 0 & 0 & 0 & 0 & 0 & 0 & 0 & 0 & 0 & 0 & 0 & 0 & 0 & 0 & 0 & 0 & 0 & 0 & 0 \\
\hline
\end{tabular}

Figure 16. Transition matrix A for Problem 1

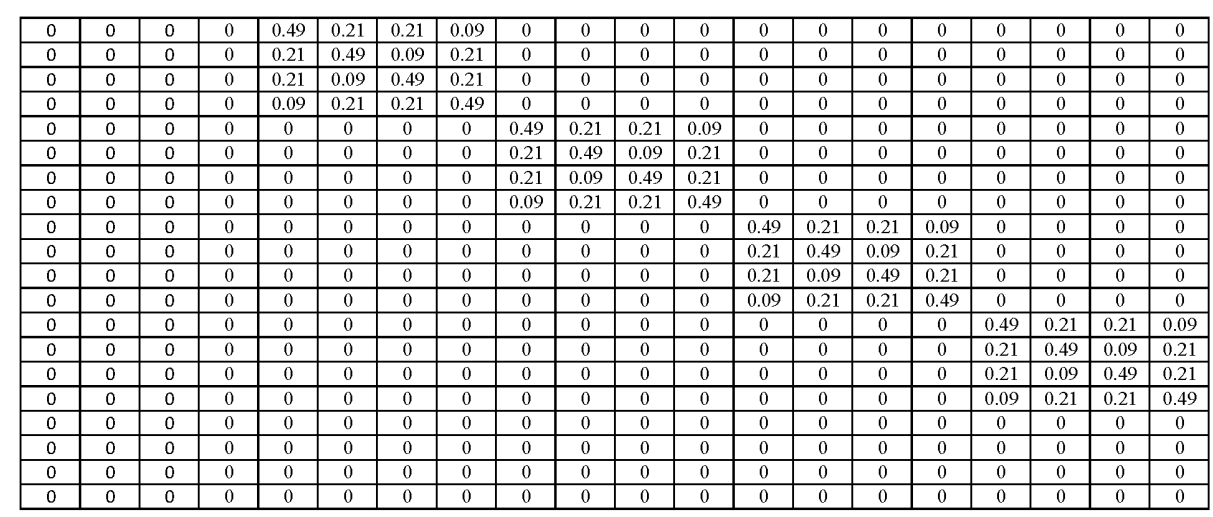

Figure 17. Transition matrix A for Problem 6 
Table 12. Heading Distribution for Problem 5

\begin{tabular}{|c|c|c|c|c|}
\hline $\begin{array}{l}\text { Time } \\
\text { Interval } \\
\qquad T_{i}\end{array}$ & $\begin{array}{c}\text { Heading } \\
h_{i j}\end{array}$ & Probability & $\begin{array}{c}\text { Distribution } \\
\text { type }\end{array}$ & Parameter \\
\hline \multirow[t]{4}{*}{$T_{1}$} & $h_{11}$ & $1 / 4$ & Gaussian & $\mu_{11}=3 \pi / 4, \sigma_{11}=\pi / 12$ \\
\hline & $h_{12}$ & $1 / 4$ & Gaussian & $\mu_{12}=\pi / 4, \sigma_{12}=\pi / 12$ \\
\hline & $h_{13}$ & $1 / 4$ & Gaussian & $\mu_{13}=-\pi / 4, \sigma_{13}=\pi / 12$ \\
\hline & $h_{14}$ & $1 / 4$ & Gaussian & $\mu_{14}=-3 \pi / 4, \sigma_{14}=\pi / 12$ \\
\hline \multirow[t]{2}{*}{$T_{2}$} & $h_{21}$ & $1 / 2$ & Gaussian & $\mu_{11}=\pi / 3, \sigma_{11}=\pi / 12$ \\
\hline & $h_{22}$ & $1 / 2$ & Gaussian & $\mu_{12}=-\pi / 3, \sigma_{12}=\pi / 12$ \\
\hline \multirow[t]{3}{*}{$T_{3}$} & $h_{31}$ & $1 / 3$ & Gaussian & $\mu_{31}=\pi / 2, \sigma_{31}=\pi / 12$ \\
\hline & $h_{32}$ & $1 / 3$ & Gaussian & $\mu_{32}=0, \sigma_{32}=\pi / 12$ \\
\hline & $h_{33}$ & $1 / 3$ & Gaussian & $\mu_{33}=-\pi / 2, \sigma_{33}=\pi / 12$ \\
\hline \multirow[t]{4}{*}{$T_{4}$} & $h_{41}$ & $1 / 4$ & Gaussian & $\mu_{41}=\pi / 6, \sigma_{41}=\pi / 24$ \\
\hline & $h_{42}$ & $1 / 4$ & Gaussian & $\mu_{42}=\pi / 12, \sigma_{42}=\pi / 24$ \\
\hline & $h_{43}$ & $1 / 4$ & Gaussian & $\mu_{43}=-\pi / 12, \sigma_{43}=\pi / 24$ \\
\hline & $h_{44}$ & $1 / 4$ & Gaussian & $\mu_{44}=-\pi / 6, \sigma_{44}=\pi / 24$ \\
\hline \multirow[t]{4}{*}{$T_{5}$} & $h_{51}$ & $1 / 4$ & Gaussian & $\mu_{41}=\pi / 4, \sigma_{41}=\pi / 16$ \\
\hline & $h_{52}$ & $1 / 4$ & Gaussian & $\mu_{42}=\pi / 8, \sigma_{42}=\pi / 16$ \\
\hline & $h_{53}$ & $1 / 4$ & Gaussian & $\mu_{43}=-\pi / 8, \sigma_{43}=\pi / 16$ \\
\hline & $h_{54}$ & $1 / 4$ & Gaussian & $\mu_{44}=\pi / 4, \sigma_{44}=\pi / 16$ \\
\hline
\end{tabular}

Table 13. Velocity Distribution for Problem 5

\begin{tabular}{|c|c|c|c|l|}
\hline $\begin{array}{c}\text { Time Interval } \\
T_{i}\end{array}$ & $\begin{array}{c}\text { Velocity } \\
\text { Distribution }\end{array}$ & Probability & $\begin{array}{c}\text { Distribution } \\
\text { type }\end{array}$ & Parameter \\
\hline \multirow{2}{*}{$T_{1}$} & $v_{11}$ & $1 / 2$ & Gaussian & $\mu_{11}=2, \sigma_{11}=0.5$ \\
\cline { 2 - 5 } & $v_{12}$ & $1 / 2$ & Gaussian & $\mu_{12}=4, \sigma_{12}=0.5$ \\
\hline \multirow{2}{*}{$T_{2}$} & $v_{21}$ & $1 / 4$ & Gaussian & $\mu_{21}=1, \sigma_{21}=0.5$ \\
\cline { 2 - 5 } & $v_{22}$ & $1 / 4$ & Gaussian & $\mu_{22}=3, \sigma_{22}=0.5$ \\
\cline { 2 - 5 } & $v_{23}$ & $1 / 4$ & Gaussian & $\mu_{23}=5, \sigma_{23}=0.5$ \\
\cline { 2 - 5 } & $v_{24}$ & $1 / 4$ & Gaussian & $\mu_{24}=7, \sigma_{24}=0.5$ \\
\hline \multirow{2}{*}{$T_{3}$} & $v_{31}$ & $1 / 3$ & Gaussian & $\mu_{31}=4, \sigma_{31}=0.5$ \\
\cline { 2 - 5 } & $v_{32}$ & $1 / 3$ & Gaussian & $\mu_{32}=6, \sigma_{32}=0.5$ \\
\cline { 2 - 5 } & $v_{33}$ & $1 / 3$ & Gaussian & $\mu_{33}=8, \sigma_{33}=0.5$ \\
\hline \multirow{2}{*}{$T_{4}$} & $v_{41}$ & $1 / 4$ & Gaussian & $\mu_{41}=3, \sigma_{41}=0.2$ \\
\cline { 2 - 5 } & $v_{42}$ & $1 / 4$ & Gaussian & $\mu_{42}=4, \sigma_{42}=0.2$ \\
\hline
\end{tabular}




\begin{tabular}{|l|l|l|l|l|}
\hline & $v_{43}$ & $1 / 4$ & Gaussian & $\mu_{43}=5, \sigma_{43}=0.2$ \\
\cline { 2 - 5 } & $v_{44}$ & $1 / 4$ & Gaussian & $\mu_{44}=6, \sigma_{44}=0.2$ \\
\hline \multirow{3}{*}{$T_{5}$} & $v_{51}$ & $1 / 4$ & Gaussian & $\mu_{51}=3, \sigma_{51}=0.1$ \\
\cline { 2 - 5 } & $v_{52}$ & $1 / 4$ & Gaussian & $\mu_{52}=4, \sigma_{52}=0.1$ \\
\cline { 2 - 5 } & $v_{53}$ & $1 / 4$ & Gaussian & $\mu_{53}=5, \sigma_{53}=0.1$ \\
\cline { 2 - 5 } & $v_{54}$ & $1 / 4$ & Gaussian & $\mu_{54}=6, \sigma_{54}=0.1$ \\
\hline
\end{tabular}

Table 14. Heading Distribution for Problem 6

\begin{tabular}{|c|c|c|c|l|}
\hline $\begin{array}{c}\text { Time Interval } \\
T_{i}\end{array}$ & $\begin{array}{c}\text { Heading } \\
h_{i j}\end{array}$ & Probability & $\begin{array}{c}\text { Distribution } \\
\text { type }\end{array}$ & Parameter \\
\hline \multirow{2}{*}{$T_{1}$} & $h_{11}$ & NA & Gaussian & $\mu_{11}=\pi / 4, \sigma_{11}=\pi / 12$ \\
\cline { 2 - 6 } & $h_{12}$ & NA & Gaussian & $\mu_{12}=-\pi / 4, \sigma_{12}=\pi / 12$ \\
\hline \multirow{2}{*}{$T_{2}$} & $h_{21}$ & NA & Gaussian & $\mu_{21}=\pi / 3, \sigma_{21}=\pi / 12$ \\
\cline { 2 - 6 } & $h_{22}$ & NA & Gaussian & $\mu_{22}=-\pi / 3, \sigma_{22}=\pi / 12$ \\
\hline \multirow{2}{*}{$T_{3}$} & $h_{31}$ & NA & Gaussian & $\mu_{31}=\pi / 2, \sigma_{31}=\pi / 12$ \\
\cline { 2 - 6 } & $h_{32}$ & NA & Gaussian & $\mu_{32}=-\pi / 2, \sigma_{32}=\pi / 12$ \\
\hline \multirow{2}{*}{$T_{4}$} & $h_{41}$ & NA & Gaussian & $\mu_{41}=\pi / 6, \sigma_{41}=\pi / 12$ \\
\cline { 2 - 6 } & $h_{42}$ & NA & Gaussian & $\mu_{42}=-\pi / 6, \sigma_{42}=\pi / 12$ \\
\hline \multirow{2}{*}{$T_{5}$} & $h_{51}$ & NA & Gaussian & $\mu_{51}=\pi / 4, \sigma_{51}=\pi / 8$ \\
\cline { 2 - 6 } & $h_{52}$ & NA & Gaussian & $\mu_{52}=-\pi / 4, \sigma_{52}=\pi / 8$ \\
\hline
\end{tabular}

Table 15. Velocity Distribution for Problem 6

\begin{tabular}{|c|c|c|c|l|}
\hline $\begin{array}{c}\text { Time Interval } \\
T_{i}\end{array}$ & $\begin{array}{c}\text { Velocity } \\
\text { Distribution }\end{array}$ & Probability & $\begin{array}{c}\text { Distribution } \\
\text { type }\end{array}$ & Parameter \\
\hline \multirow{2}{*}{$T_{1}$} & $v_{11}$ & NA & Gaussian & $\mu_{11}=2, \sigma_{11}=0.5$ \\
\cline { 2 - 5 } & $v_{12}$ & NA & Gaussian & $\mu_{12}=4, \sigma_{12}=0.5$ \\
\hline \multirow{2}{*}{$T_{2}$} & $v_{21}$ & NA & Gaussian & $\mu_{21}=2, \sigma_{21}=0.5$ \\
\cline { 2 - 5 } & $v_{22}$ & NA & Gaussian & $\mu_{22}=4, \sigma_{22}=0.5$ \\
\hline \multirow{2}{*}{$T_{3}$} & $v_{31}$ & NA & Gaussian & $\mu_{31}=6, \sigma_{31}=0.5$ \\
\cline { 2 - 5 } & $v_{32}$ & NA & Gaussian & $\mu_{32}=8, \sigma_{32}=0.5$ \\
\hline \multirow{2}{*}{$T_{4}$} & $v_{41}$ & NA & Gaussian & $\mu_{41}=3, \sigma_{41}=0.5$ \\
\cline { 2 - 5 } & $v_{42}$ & NA & Gaussian & $\mu_{42}=6, \sigma_{42}=0.5$ \\
\hline \multirow{2}{*}{$T_{5}$} & $v_{51}$ & NA & Gaussian & $\mu_{51}=4, \sigma_{51}=0.5$ \\
\cline { 2 - 5 } & $v_{52}$ & NA & Gaussian & $\mu_{52}=6, \sigma_{52}=0.5$ \\
\hline
\end{tabular}




\section{Acknowledgments}

These should be brief and placed at the end of the text before the references.

\section{References}

[1] B. Peng, S. Dang, N. Hossain and M.K. Dutta, "Shape matching and feature extraction of cross based on fourier transform and specific spatial matching", in Signal Processing and Integrated Networks (SPIN), 2015 2nd International Conference on, vol., no., (2015), pp.662-665.

[2] B. Peng, S. Dang and M.K. Dutta, "Feature detection and parameters calculation of arbitrary ellipse based on image processing technology", in Signal Processing and Integrated Networks (SPIN), 2015 2nd International Conference on , vol., no. , (2015), pp.418-420.

[3] L. R. Rabiner, "Readings in speech recognition", A. Waibel and K.-F. Lee, Eds. San Francisco, CA, USA: Morgan Kaufmann Publishers Inc., 1990, ch. A tutorial on hidden Markov models and selected applications in speech recognition, pp. 267-296. [Online]. Available: http://dl.acm.org/citation.cfm?id=108235.108253.

[4] S. Ji, R. Parr and L. Carin, "Nonmyopic multiaspect sensing with partially observable markov decision processes", Signal Processing, IEEE Transactions on, vol. 55, no. 6, (2007), pp. 2720-2730.

[5] Z. Lv, "Wearable smartphone: Wearable hybrid framework for hand and foot gesture interaction on smartphone", in Computer Vision Workshops (ICCVW), 2013 IEEE International Conference on. IEEE, (2013), pp. 436-443.

[6] Z. Lv, A. Halawani, S. Feng, H. Li and S. U. R'ehman, "Multimodal hand and foot gesture interaction for handheld devices", ACM Transactions on Multimedia Computing, Communications, and Applications (TOMM), vol. 11, no. 1s, (2014), pp. 10.

[7] S. Dang, M. Meng, D. Mathews and R. Kakimzhanov, "Control modeling and signal processing of a library self-delivery robot and its applications", in Proceedings of the 2013 International Conference on Electrical and Information Technologies for Rail Transportation (EITRT2013)-Volume II. Springer, (2014), pp. 383-390.

[8] S. Dang, J. Ju, D. Matthews, X. Feng and C. Zuo, "Efficient solar power heating system based on lenticular condensation", in Information Science, Electronics and Electrical Engineering (ISEEE), 2014 International Conference on, vol. 2, (2014), pp. 736-739.

[9] S. Dang, B. Peng, D. Matthews and A. Gholamzadeh, "Modeling and simulation of the vvvf control of the three-phase ac asynchronous electromotor based on matlab/simulink", in Information Science, Electronics and Electrical Engineering (ISEEE), 2014 International Conference on, vol. 2, (2014), pp. 746-749.

[10] S. Meyn and R. L. Tweedie, "Markov chains and stochastic stability", 2nd ed., ser. Cambridge Mathematical Library. Leiden: Cambridge Univ. Press, (2009).

[11] S. Singh and C. S. Raghavendra, "Pamas: power aware multi-access protocol with signalling for ad hoc networks", SIGCOMM Comput. Commun. Rev., vol. 28, (1998), pp. 5-26. [Online]. Available: http://doi.acm.org/10.1145/293927.293928

[12] J.-P. Laumond, P. Jacobs, M. Taix and R. Murray, "A motion planner for nonholonomic mobile robots", Robotics and Automation, IEEE Transactions on, vol. 10, no. 5, pp. $577-593$, (1994).

[13] D. Tian and N. D. Georganas, "A coverage-preserving node scheduling scheme for large wireless sensor networks", in Proceedings of the 1st ACM international workshop on Wireless sensor networks and applications, ser. WSNA '02. New York, NY, USA: ACM, (2002), pp. 32-41. [Online]. Available: http://doi.acm.org/10.1145/570738.570744.

\section{Copyright Forms}

You must include your fully-completed, signed SERSC copyright release form when you submit your paper. WE MUST HAVE THIS FORM BEFORE YOUR PAPER CAN BE PUBLISHED IN THE JOURNAL. The copyright form is available from journal home page. Authors should send their copyright forms to FAX. +82-70-7614-3027 or E-mail journal@sersc.org.

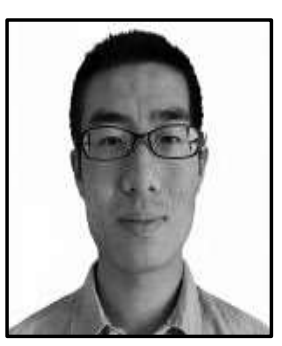

\section{Authors}

Xu Zhang, he received the BS degree from Department of Mechanical Engineering and Materials Science, University of Shanghai for Science and Technology, Shanghai, China. He is now a fourth year $\mathrm{Ph}$. D candidate at Department of MEMS, Duke 
University. His research interests include artificial intelligence, neural networks, neurorobotics.

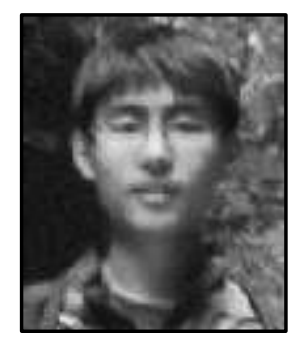

HongchuanWei, he received the BS degree from the Department of Automotive Engineering, Tsinghua University, Beijing, China. NC. His research interests include sensor network deployment and motion planning, human pilot controller design, and nonparametric Bayesian mode 